\title{
Multiple Antenna Systems with Hardware Impairments: New Performance Limits
}

\author{
Sidrah Javed, Student Member, IEEE, Osama Amin, Senior Member, IEEE, \\ Salama S. Ikki, Member, IEEE, and Mohamed-Slim Alouini, Fellow, IEEE
}

\begin{abstract}
In this paper, we accurately model the impact of aggregate hardware impairments (HWIs) in communication systems as improper Gaussian signals (IGS), which can describe the asymmetric characteristics of different HWI sources. The proposed model encourages us to adopt IGS scheme for transmission that represents a general signaling scheme compared with the conventional scheme, proper Gaussian signaling (PGS). Firstly, we express the achievable rate of multiple-input and multiple-output (MIMO) system suffering from various HWIs employing PGS and IGS schemes, when the aggregate effect of HWI is modeled as IGS. Moreover, we tune the transmit IGS statistical characteristics to maximize the achievable rate for HWI-SIMO system with two receiver combining schemes. Then, we propose an adaptive scheme to switch between maximal IGS and PGS schemes under certain conditions to improve the system performance with least computational/optimization overhead. Finally, we validate the analytic expressions through numerical and simulation results and quantify the gain reaped from adopting IGS scheme compared with PGS scheme.
\end{abstract}

Index Terms-Achievable rate, asymmetric signaling, hardware impairments, I/Q imbalance, improper Gaussian signaling, MIMO, multiple antenna, self-interference.

\section{INTRODUCTION}

The ever-increasing demand of ubiquitous high data rates with low latency and high energy efficiency are the major driving forces for the next generations of wireless communications. Immense research is carried out to develop new techniques/configurations with multiple antenna systems, extreme node densification and collaborative radio technologies to meet the expectations of the upcoming wireless networks [1]-[4]. However, the performance of the proposed systems can be affected by the non-ideal operation of radio frequency (RF) transceivers especially when the telecommunication market offers different quality products. Hardware impairments (HWIs) impose a huge challenge on next-generation network planning and deployment due to severe signal contaminations, which can degrade the overall system performance [5], [6]. For example, the deployment and operation of different types of circuitry operating at high frequencies, e.g., millimeter wave communications, can be severely limited by the non-ideal hardware behavior [7].

Copyright (c) 2015 IEEE. Personal use of this material is permitted. However, permission to use this material for any other purposes must be obtained from the IEEE by sending a request to pubs-permissions@ @ieee.org.

S. Javed, O. Amin and M.S. Alouini are with CEMSE Division, King Abdullah University of Science and Technology (KAUST), Thuwal, Makkah Province, Saudi Arabia. E-mail: \{sidrah.javed, osama.amin, slim.alouini\}@kaust.edu.sa.

S.S. Ikki is with Department of Electrical Engineering, Faculty of Engineering, Lakehead University, Ontario, Canada. E-mail: sikki@lakeheadu.ca.
HWIs arise in different RF stages such as imperfect phase shifters and mismatched local oscillators, which result in in-phase and quadrature-phase (I/Q) imbalance in the $R F$ front-end. This I/Q imbalance does not only induce phase and amplitude errors but also instigate inevitable mixing of image and desired signals. In addition, HWIs like phase noise, imperfections in analog-to-digital convertors, high power amplifier and low noise amplifier non-linearity result in additive distortion noise at the transmitter and receiver. [8], [9]. Thus, different compensation schemes and algorithms are developed to dampen the aforementioned imperfections [10], [11]. However, inaccurate HWIs modeling and parameters estimation can limit the capabilities of these schemes/algorithms [12], [13]. Hence, developing accurate impairments models play a major role in analyzing the system performance loss and proposing effective practical compensation techniques to meet the expected requirements.

\section{A. Related Work:}

Recently, several research studies analyzed the performance of wireless communication systems under different types of HWIs and proposed relevant solutions. Many studies focused on the effect of I/Q imbalance on different performance metrics such as outage probability and symbol-error rate [10], [14]-[17]. For instance, a self-interference coordination scheme was investigated in [10] to mitigate the I/Q imbalance in single-antenna multi-carrier system. In a cooperative communication setup [14], a digital baseband receiver compensation scheme was proposed for single relay systems. Moreover, an opportunistic relaying was analyzed for I/Q imbalanced multiple relay systems in [15]. In the context of a vehicularto-vehicular communications, the impact of the transceiver I/Q imbalance was analyzed using cascaded fading channel model for both single-carrier and multi-carrier systems in [16]. Furthermore, in [17] Qi and Aissa studied the bounds on average symbol error probability for the receiver I/Q imbalanced MIMO system. Different studies have presented algorithms for the joint estimation of channel gain and I/Q imbalance parameters [9], [12]. For instance, Schenk proposes a data aided approach enabled by the transmission of a preamble and algorithms for the least square channel estimation and two joint wireless channel I/Q imbalance transfer matrices based on the orthogonal and shift-orthogonal training symbols [9]. Marey et al. also proposes a novel expectation maximization algorithm based on the maximum likelihood principle to jointly estimate the channel impulse response and I/Q imbalances occurring at both the transmitter and receiver [12]. 
Different statistical models of additive hardware distortions at the transmitter and the receiver are considered in [11], [18]-[22]. In [11], Xia et al. proposed a transceiver scheme to mitigate additive distortions in full-duplex massive MIMO relaying system by exploiting the participating antenna arrays and the statistical knowledge of the channels to improve the spectral efficiency. Moreover, Björnson et al. quantified the impact of additive hardware distortions on the outage probability and suggested design guidelines for optimal performance of dual-hop relaying systems [18]. In a largescale study, Björnson et al. analyzed the energy efficiency, channel estimation and capacity limits in the presence of hardware distortions in multiple-input-single-output (MISO) and massive multiple-input-multiple-output (MIMO) systems in [19] and [20], respectively. Additionally, Björnson et al. investigated the capacity limits and multiplexing gains under physical transceiver distortions in MIMO system [21]. Furthermore, Studer et al. presented a Gaussian-distortionmodel based algorithm to dampen the residual transmit RF distortions in MIMO systems [22].

The HWIs modeling needs to consider the aggregate effect of I/Q imbalance, phase noise and distortion noises at both the transmitter and the receiver. Multiple RF front-end impairments and their individual baseband equivalent error models were analyzed in [9] and [23] . In [9], Schenk studied the modeling procedure, impact of the non-ideal hardware on the system performance, and digital compensation schemes of various RF imperfections in high data-rate wireless systems. The proposed generalized error model does not incorporate transmitter I/Q imbalances in the aggregate impairment model [9, eq. 7.6-7.8]. Similarly, Boulogeorgos et al. studied HWI impact on the energy detection spectrum sensing in cognitive radio systems [23]. However, the aforementioned research in [9] and [23] do not consider the combined effect of both transmitter and receiver I/Q mismatch. In addition, they treat the SI signal as an undesired additive distortion. Whereas, it is justified from the information theoretic definition of mutual information that treating the SI term as mere interference will result in a loss of useful information [24], [25]. Moreover, both research studies did not investigate whether the HWIs exhibit symmetric or asymmetric distortion characteristics. On the other hand, the statistical signal processing researches highlighted the asymmetric characteristics of baseband communication signals due to the I/Q imbalance [26]. Thus, throughout our research we aim to propose an accurate impairment model for the aggregate HWIs showing the asymmetric features and analyze the system performance based on the derived expressions.

\section{B. Background and Contributions:}

Asymmetric Gaussian signaling or IGS is a generalized complex Gaussian signaling scheme that relaxes the symmetric characteristics of PGS scheme allowing a correlation between the signal components and/or unequal power of each component [27]. Hence, IGS scheme offers an additional degree of design, pertinent to its circularly asymmetric characteristics. Interestingly, IGS scheme was proven to improve the system performance of different interference limited configurations such as multiple-user interference networks [28], cognitive radio systems [29], [30], full duplex relaying [31] and alternate relaying [32]. Recently, we assumed asymmetric additive distortions and studied the outage probability of SISO and single-input-multiple-output (SIMO) systems in [33].

In this paper, we consider multiple antenna systems and investigate the statistical characteristics of HWIs. In this context, we propose an accurate asymmetric statistical signal model for HWIs and analyze the achievable rate performance of different systems. Moreover, we adopt the IGS scheme to mitigate the self-interference of HWIs in order to achieve the appropriate system performance. The main contributions of this paper are summarized as follows ${ }^{1}$ :

- We propose a rigorous and precise statistical model of the aggregated hardware imperfections at different RF transmitter and receiver stages. The proposed model captures the asymmetric statistical characteristics of both the received useful signal and the accumulated HWIs based on self-interfering I/Q imbalance model.

- We express the accurate information-theoretic achievable rate based on the adopted statistical model for MIMO, SIMO with linear combiner (SIMO-LC), and SIMO with selection combiner (SIMO-SC) systems. Asymmetric transmission signaling is adopted to mitigate the effect of HWIs and achieve the appropriate system performance. Thus, we develop an optimization framework to optimally design the transmit variance and pseudo-variance in order to maximize the end-to-end achievable rate of different systems.

- We also propose an adaptive sub-optimal scheme that switches between maximal improper Gaussian signaling (MIGS) and proper Gaussian signaling transmission to reduce system complexity and optimization overhead. Moreover, we prove the necessary condition and various criterion at which the MIGS achieves better performance than its counterpart the PGS.

\section{Organization and Notations:}

The rest of the paper is organized as follows. Section II studies the statistical hardware impaired model for MIMO system followed by the system model for HWI SIMO systems with different receiver combining schemes. Section III focuses on the information-theoretic achievable rate expressions for the adopted systems. Section IV optimizes the IGS scheme to maximize the achievable rate of hardware impaired SIMO systems. As for the numerical results, Section V presents the performance degradation of various HWIs and a fair comparison between PGS and IGS based schemes. Finally, Section VI concludes the paper.

In this paper, scalars are denoted by lower-case italic letters, while vectors and matrices are denoted by boldfaced lowerand upper-case letters, respectively. For a complex scalar $x$, the conjugate and absolute value of $x$ are represented by $x^{*}$ and $|x|$, respectively. On the other hand, for a given vector

\footnotetext{
${ }^{1}$ This work was presented in part at the IEEE Global Communications Conference, Singapore, December 2017 [34]
} 
$\mathbf{x}$, the L2-norm, complex-conjugate, transpose and conjugatetranspose of $\mathbf{x}$ are represented by $\|\mathbf{x}\|_{2}, \mathbf{x}^{*}, \mathbf{x}^{\mathrm{T}}$ and $\mathbf{x}^{\mathrm{H}}$, respectively. The complex augmented random vector $\underline{\mathbf{x}}$ is defined as $\underline{\mathbf{x}}=\left[\begin{array}{ll}\mathbf{x}^{\mathrm{T}} & \mathbf{x}^{\mathrm{H}}\end{array}\right]^{\mathrm{T}}, \mathbf{x} \in \mathbb{C}^{N \times 1}$ where $\mathbb{C}^{M \times N}$ and $\mathbb{R}^{M \times N}$ describe complex-valued and real-valued matrices with dimensions $M \times N$, respectively. $\mathbb{C}_{*}^{2 N \times 1}$ represents the set of augmented vectors $\underline{\mathbf{x}}$. The identity matrix with $N$ dimension is presented by $\mathbf{I}_{N}$. For a square matrix $\mathbf{A}$, the complexconjugate, transpose, conjugate-transpose, inverse and squareroot matrix of $\mathbf{A}$ are represented by $\mathbf{A}^{*}, \mathbf{A}^{\mathrm{T}}, \mathbf{A}^{\mathrm{H}}, \mathbf{A}^{-1}$ and $\mathbf{A}^{1 / 2}$, respectively. As for the trace and determinant of $\mathbf{A}$, $\operatorname{Tr}(\mathbf{A})$ and $|\mathbf{A}|$ are used, respectively. Moreover, $\mathbf{A} \succeq 0$ and $\mathbf{A} \succ 0$ denote that $\mathbf{A}$ is a semi-definite positive matrix and positive definite matrix, respectively. The expected value operator is given by $\mathrm{E}[$.$] and the probability of occurrence of$ an event $\boldsymbol{\Omega}$ is expressed as $\operatorname{Pr}\{\boldsymbol{\Omega}\}$. The pure imaginary unit is $i$ and is defined by $i^{2}=-1$.

\section{SYSTEM DESCRIPTION}

In this section, we first introduce some statistical signal characteristics followed by the mathematical characterization of MIMO HWIs model. Various impairment sources and their effects are investigated on the transmitted and received signals. Finally, we present the transmission system model for two special scenarios; SIMO-LC and SIMO-SC.

\section{A. Statistical Signal Characteristics}

To characterize the difference between the PGS and IGS schemes, we consider a zero-mean complex Gaussian random vector $\mathbf{x} \in \mathbb{C}^{N \mathrm{x} 1}$ and introduce the following definitions:

Definition 1. The covariance and the pseudo-covariance matrices of $\mathbf{x}$ are defined as $\mathbf{C}_{\mathbf{x}} \triangleq \mathrm{E}\left[\mathbf{x x}^{\mathrm{H}}\right]$ and $\tilde{\mathbf{C}}_{\mathbf{x}} \triangleq \mathrm{E}\left[\mathbf{x x}^{\mathrm{T}}\right]$, respectively [27].

It is evident from the Definition 1 , that $\mathbf{C}_{\mathbf{x}}$ is Hermitian positive semi-definite matrix, whereas $\tilde{\mathbf{C}}_{\mathbf{x}}$ is a symmetric matrix.

Definition 2. A complex random vector $\mathrm{x}$ is called proper if its pseudo-covariance matrix $\tilde{\mathbf{C}}_{\mathbf{x}}$ vanishes (i.e., zero matrix), otherwise it is called improper [27].

Definition 3. The degree of impropriety of $x$, with variance $\sigma_{x}^{2}$ and pseudo-variance $\tilde{\sigma}_{x}^{2}$, is measured by the circularity coefficient $\kappa=\left|\tilde{\sigma}_{x}^{2}\right| / \sigma_{x}^{2}$, where $0 \leq \kappa \leq 1, \kappa=0$ denotes proper signal and $\kappa=1$ denotes maximally improper signal [35].

According to Definitions 1-3, the complex Gaussian random variables can be fully characterized using both the covariance and pseudo-covariance matrices. Therefore, a complex-Gaussian random variable $\mathbf{x}$ is fully described as $\mathcal{C N}\left(\mu_{\mathbf{x}}, \mathbf{C}_{\mathbf{x}}, \tilde{\mathbf{C}}_{\mathbf{x}}\right)$ where $\mu_{\mathbf{x}}$ is the mean of $\mathbf{x}$.

\section{B. MIMO Hardware Impairment}

In this subsection, we mathematically express the received signal of the HWI MIMO system. As such, we first consider the ideal scenario of MIMO transceivers with no HWI and use

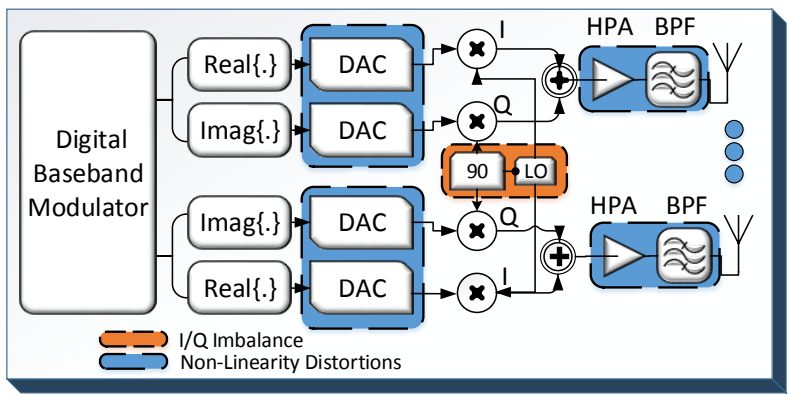

Fig. 1: Hardware impaired MIMO transmitter RF front-end

it as a benchmark to the system with imperfect hardware. We assume a MIMO system employing $N_{\mathrm{T}}$ RF transmit branches to communicate with a destination that uses $N_{\mathrm{R}}$ RF receiver branches through independent wireless fading channels.

1) Ideal MIMO System: Consider the transmission of a complex circularly symmetric information vector $\mathbf{x} \in \mathbb{C}^{N_{\mathrm{T}} \mathrm{x}}$ with $\mathbf{x} \sim \mathcal{C N}\left(\mathbf{0}, \mathbf{C}_{\mathbf{x}}, \mathbf{0}\right)$. Assume $\mathbf{x}$ bears the transmit power that is attained after the power amplifier ${ }^{2}$. The time-domain received signal vector for the ideal MIMO system is then expressed as

$$
\mathbf{y}_{\mathrm{id}}=\mathbf{H} \mathbf{x}+\mathbf{w},
$$

where $\mathbf{H} \in \mathbb{C}^{N_{\mathrm{R}} \mathrm{x} N_{\mathrm{T}}}$ is the slowly-varying flat-fading channel matrix and each element in $\mathbf{H}\left(h_{i j}, i=1, \cdots, N_{\mathrm{R}}, j=\right.$ $\left.1, \cdots, N_{\mathrm{T}}\right)$ is independent and identically distributed (i.i.d.) complex normal random variable; $h_{i j} \sim \mathcal{C N}(0, \pi, 0)$, and $\mathbf{w} \in \mathbb{C}^{N_{\mathrm{R}} \times 1}$ is the additive proper white complex Gaussian noise (AWGN) with i.i.d. random variables $w_{i} \sim$ $\mathcal{C N}\left(0, \sigma_{w}^{2}, 0\right)$, exhibiting diagonal covariance matrix $\mathbf{C}_{\mathbf{w}}$.

2) Non-Ideal MIMO System: The RF transceiver impairments cannot be modeled merely as an additive noise because of their distinct behavior. To develop a statistical mathematical model for the accumulated HWIs, we consider a MIMO RF front-end direct-conversion based architecture for spatially diverse transmitter and receiver as shown in Fig. 1 and Fig. 2, respectively. At the transmitter side in Fig. 1, the real and imaginary parts of information signal $\mathbf{x}$ pass through digital-to-analog converter and then up-convert to the desired carrier frequency using homodyne architecture which introduces HWIs. The mismatched local-oscillator and phase shifter introduce phase and amplitude errors, commonly referred as I/Q imbalance, which result in limited image rejection exhibiting self-inference. Based on the practically validated I/Q mismatch model presented in [9, eq. 5.22], [36, eq. 1] and [10, eq. 8], the equivalent quadrature imbalanced base-band transmit signal is given as

$$
\mathbf{x}_{1}=\mathbf{V}_{1} \mathbf{x}+\mathbf{V}_{2} \mathbf{x}^{*}
$$

where $\mathbf{V}_{1} \in \mathbb{C}^{N_{\mathrm{T}} \times N_{\mathrm{T}}}$ and $\mathbf{V}_{2} \in \mathbb{C}^{N_{\mathrm{T}} \times N_{\mathrm{T}}}$ capture the amplitude and rotational errors

$$
\begin{aligned}
& \mathbf{V}_{1}=\left(\mathbf{I}_{N_{\mathrm{T}}}+\mathbf{A}_{\mathrm{T}} e^{j \boldsymbol{\Theta}_{\mathrm{T}}}\right) / 2, \\
& \mathbf{V}_{2}=\mathbf{I}_{N_{\mathrm{T}}}-\mathbf{V}_{1}^{*}=\left(\mathbf{I}_{N_{\mathrm{T}}}-\mathbf{A}_{\mathrm{T}} e^{-j \boldsymbol{\Theta}_{\mathrm{T}}}\right) / 2 .
\end{aligned}
$$

\footnotetext{
${ }^{2}$ Signal power notation is omitted for the sake of simplicity and clarity.
} 


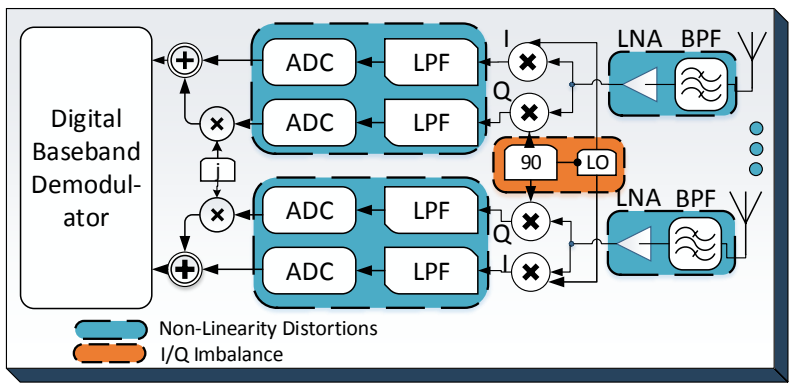

Fig. 2: Hardware impaired MIMO receiver RF front-end [33]

The $\mathbf{A}_{\mathrm{T}}$ and $\boldsymbol{\Theta}_{\mathrm{T}}$ matrices in (3) and (4) are diagonal matrices incorporating amplitude and phase errors from each transmitter branch, respectively [9, eq. 5.23, 5.24]. These parameters can be jointly estimated with channel gain using different algorithms [9], [12].The image rejection ratio (IRR) of the I/Q mixer stage of the $k^{\text {th }}$ transmitter stream is defined as $\Upsilon_{\mathrm{t}, k}=\frac{\left|\nu_{k 1}\right|^{2}}{\left|\nu_{k 2}\right|^{2}}$, where $\nu_{k 1}$ and $\nu_{k 2}$ are $k^{\text {th }}$ diagonal elements of $\mathbf{V}_{1}$ and $\mathbf{V}_{2}$, respectively [10], [23]. Transmitter with perfect $\mathrm{I} / \mathrm{Q}$ balance exhibits $\mathbf{V}_{1}=\mathrm{I}$ and $\mathbf{V}_{2}=\mathbf{0}$.

Moreover, non-linear transfer function of various transceiver stages, such as digital-to-analog converters, high power amplifier and band-pass filters, results in additive distortion $\mathbf{d}_{\mathrm{T}} \in \mathbb{C}^{N_{\mathrm{T}} \times 1}$ in the respective transceiver branch raising the noise floor. It is modeled as $\mathbf{d}_{\mathrm{T}} \sim \mathcal{C N}\left(\mathbf{0}, \mathbf{C}_{\mathrm{T}}, \mathbf{0}\right)$ [18, eq. 3], [9, eq. 7.9], such that the transmitted signal reduces to

$$
\begin{aligned}
\mathbf{x}_{\mathrm{tx}} & =\mathbf{x}_{1}+\mathbf{d}_{\mathrm{T}} \\
& =\mathbf{V}_{1} \mathbf{x}+\mathbf{V}_{\mathbf{2}} \mathbf{x}^{*}+\mathbf{d}_{\mathrm{T}} .
\end{aligned}
$$

The impaired transmitted signal undergoes channel fading and receiver distortions $\mathbf{d}_{\mathrm{R}} \in \mathbb{C}^{N_{\mathrm{R}} \times 1}$ from low noise amplifier, band pass filter, image rejection filters, analog to digital converter and thermal noise. Therefore, the received signal vector $\mathbf{y}_{\mathrm{rx}}$ with additive receiver distortion $\mathbf{d}_{\mathrm{R}} \sim \mathcal{C N}\left(\mathbf{0}, \mathbf{C}_{\mathrm{R}}, \mathbf{0}\right)$ [18] is given by

$$
\mathbf{y}_{\mathrm{rx}}=\mathbf{H x}_{\mathrm{tx}}+\mathbf{d}_{\mathrm{R}} .
$$

Similar to the transmitter I/Q imbalance, the local oscillator, phase shifters and mixers also introduce $\mathrm{I} / \mathrm{Q}$ imbalance at receiver, which is expressed as [9, eq. 5.28] and [36, eq. 3].

$$
\mathbf{y}=\boldsymbol{\Gamma}_{1} \mathbf{y}_{\mathrm{rx}}+\boldsymbol{\Gamma}_{2} \mathbf{y}_{\mathrm{rx}}^{*}
$$

where $\Gamma_{1} \in \mathbb{C}^{N_{\mathrm{R}} \times N_{\mathrm{R}}}$ and $\boldsymbol{\Gamma}_{2} \in \mathbb{C}^{N_{\mathrm{R}} \times N_{\mathrm{R}}}$ are defined, respectively, as [9]

$$
\begin{aligned}
& \boldsymbol{\Gamma}_{1}=\left(\mathbf{I}_{N_{\mathrm{R}}}+\mathbf{A}_{\mathrm{R}} e^{j \Theta_{\mathrm{R}}}\right) / 2, \\
& \boldsymbol{\Gamma}_{2}=\mathbf{I}_{N_{\mathrm{R}}}-\boldsymbol{\Gamma}_{1}^{*}=\left(\mathbf{I}_{N_{\mathrm{R}}}-\mathbf{A}_{\mathrm{R}} e^{-j \Theta_{\mathrm{R}}}\right) / 2,
\end{aligned}
$$

The diagonal matrices $\mathbf{A}_{\mathrm{R}}$ and $\Theta_{\mathrm{R}}$ capture the amplitude and rotational imbalances for each receiver stream, respectively. The I/Q mixer stage of $k^{\text {th }}$ receiver stream exhibits IRR $\Upsilon_{\mathrm{r}, k}=\frac{\left|\mu_{k 1}\right|^{2}}{\left|\mu_{k}\right|^{2}}$, where $\mu_{k 1}$ and $\mu_{k 2}$ are $k^{\text {th }}$ diagonal elements of $\boldsymbol{\Gamma}_{1}$ and $\boldsymbol{\Gamma}_{2}$, respectively.

\section{Asymmetric Hardware Impairments Statistical Model}

To characterize the HWIs, we present the mathematical model for down-converted time-domain single-carrier received signal with aggregated HWIs in Lemma 1. We further analyze asymmetric characteristics of the aggregated distortions and the received signal under PGS and IGS transmit schemes. ${ }^{3}$

Lemma 1. The aggregate statistical mathematical model that encompasses the self-interfering received signal due to the HWIs at the transmitter and the receiver sides over a flat fading channel $\mathbf{H} \in \mathbb{C}^{N_{\mathrm{R}} \times N_{\mathrm{T}}}$ is given by

$$
\mathbf{y}=\overline{\mathbf{H}}_{1} \mathbf{x}+\overline{\mathbf{H}}_{2} \mathbf{x}^{*}+\mathbf{z},
$$

where $\mathbf{z}$ owns asymmetric characteristics with $\mathbf{z} \sim$ $\mathcal{C N}\left(\mathbf{0}, \mathbf{C}_{\mathbf{z}}, \tilde{\mathbf{C}}_{\mathbf{z}}\right), \quad \overline{\mathbf{H}}_{1}=\boldsymbol{\Gamma}_{1} \mathbf{H} \mathbf{V}_{1}+\boldsymbol{\Gamma}_{2} \mathbf{H}^{*} \mathbf{V}_{2}^{*}$ and $\overline{\mathbf{H}}_{2}=$ $\boldsymbol{\Gamma}_{1} \mathbf{H V}_{2}+\boldsymbol{\Gamma}_{2} \mathbf{H}^{*} \mathbf{V}_{1}^{*}$ with $\overline{\mathbf{H}}_{1}, \overline{\mathbf{H}}_{2} \in \mathbb{C}^{N_{\mathrm{R}} \times N_{\mathrm{T}}}$.

Proof. By substituting (5) and (6) in (7), we obtain

$$
\mathbf{y}=\overline{\mathbf{H}}_{1} \mathbf{x}+\overline{\mathbf{H}}_{2} \mathbf{x}^{*}+\boldsymbol{\Gamma}_{1}\left(\mathbf{H d}_{\mathrm{T}}+\mathbf{d}_{\mathrm{R}}\right)+\boldsymbol{\Gamma}_{2}\left(\mathbf{H}^{*} \mathbf{d}_{\mathrm{T}}^{*}+\mathbf{d}_{\mathrm{R}}^{*}\right),
$$

which results in $\mathbf{z}=\boldsymbol{\Gamma}_{1}\left(\mathbf{H d}_{\mathrm{T}}+\mathbf{d}_{\mathrm{R}}\right)+\boldsymbol{\Gamma}_{2}\left(\mathbf{H}^{*} \mathbf{d}_{\mathrm{T}}^{*}+\mathbf{d}_{\mathrm{R}}^{*}\right)$. To prove that $\mathbf{z}$ is IGS, we evaluate the pseudo-covariance that is derived to be

$$
\tilde{\mathbf{C}}_{\mathbf{z}}=\boldsymbol{\Gamma}_{1}\left(\mathbf{H} \mathbf{C}_{\mathrm{T}} \mathbf{H}^{\mathrm{H}}+\mathbf{C}_{\mathrm{R}}\right) \boldsymbol{\Gamma}_{2}^{\mathrm{T}}+\boldsymbol{\Gamma}_{2}\left(\mathbf{H}^{*} \mathbf{C}_{\mathrm{T}} \mathbf{H}^{\mathrm{T}}+\mathbf{C}_{\mathrm{R}}\right) \boldsymbol{\Gamma}_{1}^{\mathrm{T}} .
$$

The non-zero $\tilde{\mathbf{C}}_{\mathbf{z}}$ indicates the asymmetric characteristics of $\mathbf{z}$. Moreover, the aggregate noise covariance can be evaluated as

$$
\mathbf{C}_{\mathbf{z}}=\boldsymbol{\Gamma}_{1}\left(\mathbf{H C}_{\mathrm{T}} \mathbf{H}^{\mathrm{H}}+\mathbf{C}_{\mathrm{R}}\right) \boldsymbol{\Gamma}_{1}^{\mathrm{H}}+\boldsymbol{\Gamma}_{2}\left(\mathbf{H}^{*} \mathbf{C}_{\mathrm{T}} \mathbf{H}^{\mathrm{T}}+\mathbf{C}_{\mathrm{R}}\right) \boldsymbol{\Gamma}_{2}^{\mathrm{H}} .
$$

It is crucial to note that the HWI received signal model in (10) reduces to (1) under ideal hardware assumptions, i.e., when $\Gamma_{1}=V_{1}=\mathbf{I}, \Gamma_{2}=V_{2}=\mathbf{0}, \mathbf{d}_{\mathrm{T}}=\mathbf{0}$ and $\mathbf{d}_{\mathrm{R}}=\mathbf{w}$. The asymmetric characteristics of $\mathbf{z}$ result from the widely linear transformation structure applied on $\mathbf{d}_{\mathrm{R}}$ and $\mathbf{d}_{\mathrm{T}}$. It is interesting to observe that the information signal in $\mathbf{y}$ also undergoes self-interference. Thus, the HWIs convert the symmetric input signals to asymmetric signals. Therefore, the statistical signal characteristics are worth investigating with PGS or IGS information signal $\mathrm{x}$.

1) $P G S$ Transmission: Assuming the information signal $\mathbf{x} \sim \mathcal{C N}\left(\mathbf{0}, \mathbf{C}_{\mathbf{x}}, \mathbf{0}\right)$, aggregate HWIs $\mathbf{z} \sim \mathcal{C N}\left(\mathbf{0}, \mathbf{C}_{\mathbf{z}}, \widetilde{\mathbf{C}}_{\mathbf{z}}\right)$ given by Lemma 1 and the perfect knowledge of CSI and HWIs coefficients, the received signal covariance $\mathbf{C}_{\mathbf{y}_{\mathrm{P}}}$ and pseudo-covariance $\tilde{\mathbf{C}}_{\mathbf{y}_{\mathrm{P}}}$ are expressed, respectively, as

$$
\begin{aligned}
\mathbf{C}_{\mathbf{y}_{\mathrm{P}}} & =\overline{\mathbf{H}}_{1} \mathbf{C}_{\mathbf{x}} \overline{\mathbf{H}}_{1}^{\mathrm{H}}+\overline{\mathbf{H}}_{2} \mathbf{C}_{\mathbf{x}} \overline{\mathbf{H}}_{2}^{\mathrm{H}}+\mathbf{C}_{\mathbf{z}} \\
\tilde{\mathbf{C}}_{\mathbf{y}_{\mathrm{P}}} & =\overline{\mathbf{H}}_{1} \mathbf{C}_{\mathbf{x}} \overline{\mathbf{H}}_{2}^{\mathrm{T}}+\overline{\mathbf{H}}_{2} \mathbf{C}_{\mathbf{x}} \overline{\mathbf{H}}_{1}^{\mathrm{T}}+\tilde{\mathbf{C}}_{\mathbf{z}},
\end{aligned}
$$

\footnotetext{
${ }^{3}$ It is important to highlight that this work focuses on the narrowband/singlecarrier MIMO system for the sake of brevity and simplicity. However, the same impairment model can be straight forwardly extended to the wideband/multicarrier MIMO systems to capture the effect of different impairments. In such systems, the rotation of the desired signals caused by the phase noise is equal for all carriers, therefore it is termed as the common phase error and can be accommodated in the multiplicative I/Q parameters. Furthermore, the intercarrier interference due to phase noise and the leakage from mirror sub-carrier signal caused by the I/Q imbalance can be accumulated in the distortion terms $\mathbf{d}_{\mathrm{T}}$ and $\mathbf{d}_{\mathrm{R}}$ at the transmitter and receiver respectively.
} 


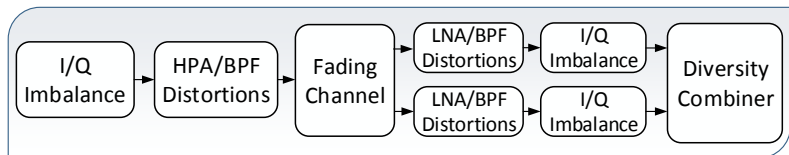

Fig. 3: Hardware impaired SIMO block diagram

2) IGS Transmission: The transmitted signal is characterized by $\mathbf{x} \sim \mathcal{C N}\left(\mathbf{0}, \mathbf{C}_{\mathbf{x}}, \tilde{\mathbf{C}}_{\mathbf{x}}\right)$. The covariance $\mathbf{C}_{\mathbf{y}_{\mathrm{I}}}$ and the pseudo-covariance $\tilde{\mathbf{C}}_{\mathbf{y}_{\mathrm{I}}}$ are given, respectively, by

$$
\begin{aligned}
\mathbf{C}_{\mathbf{y}_{\mathrm{I}}} & =\mathbf{C}_{\mathbf{y}_{\mathrm{P}}}+\overline{\mathbf{H}}_{1} \tilde{\mathbf{C}}_{\mathbf{x}} \overline{\mathbf{H}}_{2}^{\mathrm{H}}+\overline{\mathbf{H}}_{2} \tilde{\mathbf{C}}_{\mathbf{x}}^{\mathrm{H}} \overline{\mathbf{H}}_{1}^{\mathrm{H}} \\
\tilde{\mathbf{C}}_{\mathbf{y}_{\mathrm{I}}} & =\tilde{\mathbf{C}}_{\mathbf{y}_{\mathrm{P}}}+\overline{\mathbf{H}}_{1} \tilde{\mathbf{C}}_{\mathbf{x}} \overline{\mathbf{H}}_{1}^{\mathrm{T}}+\overline{\mathbf{H}}_{2} \tilde{\mathbf{C}}_{\mathbf{x}}^{\mathrm{H}} \overline{\mathbf{H}}_{2}^{\mathrm{T}} .
\end{aligned}
$$

Therefore, the resultant received signal at MIMO receiver under both PGS and IGS transmission schemes exhibits asymmetric characteristics as deduced from the non-zero pseudocovariance matrices in (15) and (17), respectively. Moreover, employing IGS transmission introduces another design flexibility that can reduce the resultant HWIs impact and improve the system performance.

\section{Receiver Combining Techniques of SIMO Systems}

Different receiver diversity techniques can be adopted in SIMO with $N_{\mathrm{R}}$ receiver streams to achieve performance gain and combat fading. We consider two such techniques namely linear combining and selection combining. Following the block architectural layout of impairment blocks as shown in Fig 3, the impaired transmit signal is given by

$$
x_{\mathrm{SIMO}}=\nu_{1} x+\nu_{2} x^{*}+d_{\mathrm{T}},
$$

where, the I/Q amplitude and phase error impact at transmitter are captured in $\nu_{1}$ and $\nu_{2}$, as given in (3) and (4), respectively, with $N_{\mathrm{T}}=1$. Moreover, the aggregate distortion noise from various blocks is assumed to be $d_{\mathrm{T}} \in \mathbb{C}$ [9]. Using (10), the down-converted received signal vector $\mathbf{y}$ contains HWIs from various blocks in unit transmitter and multireceiver streams. In linear combining diversity scheme, all observations are superposed in $y_{\text {SIMO }}$ using linear combining vector $\phi \in \mathbb{C}^{N_{\mathrm{R}} \times 1}$ as

$$
y_{\text {SIMO }}=\phi^{H} \mathbf{y}=\phi^{\mathrm{H}} \overline{\mathbf{h}}_{1} x+\phi^{\mathrm{H}} \overline{\mathbf{h}}_{2} x^{*}+\phi^{\mathrm{H}} \hat{\mathbf{z}},
$$

$\overline{\mathbf{h}}_{1}, \overline{\mathbf{h}}_{2}$ and $\hat{\mathbf{z}} \in \mathbb{C}^{N_{\mathrm{R}} \times 1}$ can be derived by imposing $N_{\mathrm{T}}=1$ in Lemma 1. The signal-to-distortion plus noise ratio is given by

$$
\operatorname{SDNR}=\frac{\phi^{\mathrm{H}} \boldsymbol{\Delta}_{\mathrm{m}} \phi}{\phi^{\mathrm{H}} \mathbf{C}_{\mathbf{z}} \phi},
$$

where $\boldsymbol{\Delta}_{1}=\phi^{\mathrm{H}}\left(\sigma_{x}^{2} \overline{\mathbf{h}}_{1} \overline{\mathbf{h}}_{1}^{\mathrm{H}}+\sigma_{x}^{2} \overline{\mathbf{h}}_{2} \overline{\mathbf{h}}_{2}^{\mathrm{H}}\right) \phi$ and $\boldsymbol{\Delta}_{2}=$ $\phi^{\mathrm{H}}\left(\sigma_{x}^{2} \overline{\mathbf{h}}_{1} \overline{\mathbf{h}}_{1}^{\mathrm{H}}+\sigma_{x}^{2} \overline{\mathbf{h}}_{2} \overline{\mathbf{h}}_{2}^{\mathrm{H}}+\tilde{\sigma}_{x}^{2} \overline{\mathbf{h}}_{1} \overline{\mathbf{h}}_{2}^{\mathrm{H}}+\tilde{\sigma}_{x}^{* 2} \overline{\mathbf{h}}_{2} \overline{\mathbf{h}}_{1}^{\mathrm{H}}\right) \phi$, for PGS and IGS transmission, respectively. Assuming invertible $\mathbf{C}_{\mathbf{z}}$, the receiver linear combining vector $\phi$, which maximizes SDNR, is the eigen-vector of $\mathbf{C}_{\mathbf{z}}{ }^{-1} \boldsymbol{\Delta}_{m}$ corresponding to the maximum eigenvalue of the product $\mathbf{C}_{\mathbf{z}}{ }^{-1} \boldsymbol{\Delta}_{m}$.

To reduce the receiver complexity and computation overhead, and avoid antenna synchronization problems, selection combining scheme is adopted [37]. In this scheme, receiver selects the receiver branch that achieves the best performance to detect the transmitted signal. Therefore, the selected receiver branch can be chosen to improve different performance metrics such as instantaneous achievable rate, system outage probability, signal-to-noise ratio (SNR) and energy efficiency.

\section{AChievable Rate}

In this section, we derive the achievable rate of non-ideal hardware MIMO system, then we consider special scenarios of SIMO-LC and SIMO-SC systems. Under the assumption of proper Gaussian additive noise, the achievable rate is maximized when the transmitted signal is chosen to be PGS [26]. However, when the equivalent noise is IGS, the transmitted signal should be chosen from IGS scheme to outperform the PGS transmit scheme. Therefore, hardware impaired systems should adopt and optimize IGS scheme due to the asymmetric characteristics of HWI self-interference. Adopting IGS is expected to dampen the effects of HWIs and improve the achievable rate gain. The achievable rate (bits/channel use) in terms of mutual information between complex Gaussian transmitted random variable $\mathrm{x}$ and the received signal vector $\mathbf{y}$ in the presence of Gaussian interference $\mathbf{z}$, is given as

$$
R=I(\mathbf{x} ; \mathbf{y})=h(\mathbf{y})-h(\mathbf{y} \mid \mathbf{x})=h(\mathbf{y})-h(\mathbf{z}),
$$

where, $I(\mathbf{x} ; \mathbf{y})$ is mutual information between two variables $\mathbf{x}$ and $\mathbf{y}$ and $h($.$) is the differential entropy of the information$ signal that is given by the following lemma.

Lemma 2. The entropy of an IGS random variable $\mathrm{x} \in \mathbb{C}^{N \times 1}$, with augmented covariance matrix $\underline{\mathbf{C}}_{\mathrm{x}}$, is in general a function of both covariance and pseudo-covariance matrices and is given as [26]

$$
h(\mathbf{x})=\frac{1}{2} \log _{2}\left((\pi e)^{2 \mathrm{~N}}\left|\underline{\mathbf{C}}_{\mathbf{x}}\right|\right),
$$

where $\underline{\mathbf{C}}_{\mathrm{x}}$ is defined as

$$
\underline{\mathbf{C}}_{\mathbf{x}} \triangleq \mathrm{E}\left(\left[\begin{array}{c}
\mathbf{x} \\
\mathbf{x}^{*}
\end{array}\right]\left[\begin{array}{c}
\mathbf{x} \\
\mathbf{x}^{*}
\end{array}\right]^{\mathrm{H}}\right)=\left[\begin{array}{cc}
\mathbf{C}_{\mathbf{x}} & \tilde{\mathbf{C}}_{\mathbf{x}} \\
\tilde{\mathbf{C}}_{\mathbf{x}}^{*} & \mathbf{C}_{\mathbf{x}}^{*}
\end{array}\right] .
$$

For a PGS random variable $\mathbf{x}$ with $\tilde{\mathbf{C}}_{\mathbf{x}}=0$, the entropy reduces to the well-known expression $h(\mathbf{x})=$ $\log _{2}\left((\pi e)^{\mathrm{N}}\left|\mathbf{C}_{\mathbf{x}}\right|\right)$.

Lemma 3. Hermitian positive semi-definite property of covariance matrix $\mathbf{C}_{\mathbf{x}}$, symmetry property of pseudo-covariance matrix $\tilde{\mathbf{C}}_{\mathbf{x}}$ and Schur complement renders the determinant of augmented covariance matrix $\underline{\mathbf{C}}_{\mathbf{x}}$ as

$$
\left|\underline{\mathbf{C}}_{\mathbf{x}}\right|=\left|\mathbf{C}_{\mathbf{x}}\right|^{2}\left|\mathrm{I}-\mathbf{C}_{\mathbf{x}}{ }^{-1} \tilde{\mathbf{C}}_{\mathbf{x}} \mathbf{C}_{\mathbf{x}}{ }^{-\mathrm{T}}{\tilde{\mathbf{C}_{\mathbf{x}}}}^{\mathrm{H}}\right| .
$$

Proof. Proof is given in Appendix A.

\section{A. Hardware Impaired MIMO}

In this section, we analyze the achievable rate for the cases of ideal RF front-end hardware followed by hardware impaired RF front-end transceiver branches. 
1) Ideal Scenario: In the absence of any HWI distortion, the only undesired signal $\mathbf{z}$ is the AWGN thermal noise $\mathbf{w}$ at the receiver branches, which motivates us to adopt PGS scheme for the transmission. Therefore, based on Lemma 2, the entropy of the received signal and the AWGN noise are expressed, respectively, as

$$
\begin{aligned}
& h(\mathbf{y})=\log _{2}\left((\pi e)^{N_{\mathrm{R}}}\left|\mathbf{C}_{\mathbf{y}}\right|\right), \\
& h(\mathbf{w})=\log _{2}\left((\pi e)^{N_{\mathrm{R}}}\left|\mathbf{C}_{\mathbf{w}}\right|\right),
\end{aligned}
$$

where $\mathbf{C}_{\mathbf{y}}$ and $\mathbf{C}_{\mathbf{w}}$ are found to be $\mathbf{C}_{\mathbf{y}}=\mathbf{H C}_{\mathbf{x}} \mathbf{H}^{\mathrm{H}}+\mathbf{C}_{\mathbf{w}}$ and $\mathbf{C}_{\mathbf{w}}=\sigma_{w}^{2} \mathbf{I}_{N_{\mathrm{R}}}$ respectively. Therefore, the achievable rate of ideal hardware MIMO system is found from (21) under the assumption of i.i.d. transmitted signals with covariance $\mathbf{C}_{\mathbf{x}}=\sigma_{x}^{2} \mathbf{I}_{N_{\mathrm{T}}}$ as

$$
R_{\mathrm{MIMO}-\text { Ideal }}=B \log _{2}\left|\mathbf{I}_{N_{\mathrm{R}}}+\frac{\sigma_{x}^{2}}{\sigma_{w}^{2}} \mathbf{H H}^{\mathrm{H}}\right| .
$$

where $B$ is the bandwidth of the channel. Thus, $R_{\mathrm{MIMO} \text {-Ideal }}$ (bits/sec) can also be presented in terms of the eigen values $\lambda_{k}$ of the unnormalized Wishart matrix $\mathbf{H}^{\mathrm{H}} \mathbf{H}$ as

$$
R_{\text {MIMO-Ideal }}=\sum_{k=1}^{\min \left(N_{\mathrm{R}}, N_{\mathrm{T}}\right)} B \log _{2}\left(1+\frac{\sigma_{x}^{2}}{\sigma_{w}^{2}} \lambda_{k}\right) .
$$

2) Non-Ideal Hardware under PGS transmission: For practical non-ideal transceiver hardware that suffers from various HWIs modeled in (10), the improper undesired signal $\mathbf{z}$ is a composite of the aggregated I/Q imbalance and distortion noises at the transceiver branches. The covariance and pseudocovariance matrices of the improper HWIs are given by (13) and (12), respectively. Under the assumption of PGS transmission, the covariance $\left(\mathbf{C}_{\mathbf{y}_{\mathrm{P}}}\right)$ and pseudo-covariance $\left(\tilde{\mathbf{C}}_{\mathbf{y}_{\mathrm{P}}}\right)$ matrices of the received signal $\mathbf{y}$ are given by (14) and (15), respectively. Furthermore, it is evident from (13) and (14) that $\mathbf{C}_{\mathbf{z}}$ and $\mathbf{C}_{\mathbf{y}}$ are non-singular. Using Lemma 2 and Lemma 3, the differential entropy of the received signal $\mathbf{y}$ and the improper undesired signal $\mathbf{z}$ is given as

$h(\mathbf{y})=B \log _{2}\left((\pi e)^{N}\left|\mathbf{C}_{\mathbf{y}_{\mathrm{P}}}\right|\right)+\frac{B}{2} \log _{2}\left|\mathbf{I}_{N_{\mathrm{R}}}-\mathbf{C}_{\mathbf{y}_{\mathrm{P}}}^{-1} \tilde{\mathbf{C}}_{\mathbf{y}_{\mathrm{P}}} \mathbf{C}_{\mathbf{y}_{\mathrm{P}}}^{-\mathrm{T}} \tilde{\mathbf{C}}_{\mathbf{y}_{\mathrm{P}}}^{\mathrm{H}}\right|$ $h(\mathbf{z})=B \log _{2}\left((\pi e)^{N}\left|\mathbf{C}_{\mathbf{z}}\right|\right)+\frac{B}{2} \log _{2}\left|\mathbf{I}_{N_{\mathrm{R}}}-\mathbf{C}_{\mathbf{z}}^{-\mathbf{1}} \tilde{\mathbf{C}}_{\mathbf{z}} \mathbf{C}_{\mathbf{z}}^{-\mathrm{T}} \tilde{\mathbf{C}}_{\mathbf{z}}^{\mathrm{H}}\right|$.

Therefore, the achievable rate is given by

$R_{\mathrm{MIMO}-\mathrm{PGS}}=B \log _{2} \frac{\left|\mathbf{C}_{\mathbf{y}_{\mathrm{P}}}\right|}{\left|\mathbf{C}_{\mathbf{z}}\right|}+\frac{B}{2} \log _{2} \frac{\left|\mathbf{I}_{N_{\mathrm{R}}}-\mathbf{C}_{\mathbf{y}_{\mathrm{P}}}^{-1} \tilde{\mathbf{C}}_{\mathbf{y}_{\mathrm{P}}} \mathbf{C}_{\mathbf{y}_{\mathrm{P}}}^{-\mathrm{T}} \tilde{\mathbf{C}}_{\mathbf{y}_{\mathrm{P}}}^{\mathrm{H}}\right|}{\left|\mathbf{I}_{N_{\mathrm{R}}}-\mathbf{C}_{\mathbf{z}}^{-1} \tilde{\mathbf{C}}_{\mathbf{z}} \mathbf{C}_{\mathbf{z}}^{-\mathrm{T}} \tilde{\mathbf{C}}_{\mathbf{z}}^{\mathrm{H}}\right|}$.

3) Non-Ideal Hardware under IGS transmission: Similarly, the achievable rate expression can be obtained using the mutual information between $\mathbf{y}$ and $\mathbf{z}$ assuming HWIs and with the employment of IGS for transmission as

$R_{\mathrm{MIMO}-\mathrm{IGS}}=B \log _{2} \frac{\left|\mathbf{C}_{\mathbf{y}_{\mathrm{I}}}\right|}{\left|\mathbf{C}_{\mathbf{z}}\right|}+\frac{B}{2} \log _{2} \frac{\left|\mathbf{I}_{N_{\mathrm{R}}}-\mathbf{C}_{\mathbf{y}_{\mathrm{I}}}^{-1} \tilde{\mathbf{C}}_{\mathbf{y}_{\mathrm{I}}} \mathbf{C}_{\mathbf{y}_{\mathrm{I}}}^{-\mathrm{T}} \tilde{\mathbf{C}}_{\mathbf{y}_{\mathrm{I}}}^{\mathrm{H}}\right|}{\left|\mathbf{I}_{N_{\mathrm{R}}}-\mathbf{C}_{\mathbf{z}}^{-1} \tilde{\mathbf{C}}_{\mathbf{z}} \mathbf{C}_{\mathbf{z}}^{-\mathrm{T}} \tilde{\mathbf{C}}_{\mathbf{z}}^{\mathrm{H}}\right|}$. where, the covariance $\left(\mathbf{C}_{\mathbf{y}_{\mathbf{I}}}\right)$ and pseudo-covariance $\left(\tilde{\mathbf{C}}_{\mathbf{y}_{\mathbf{I}}}\right)$ matrices of the received signal $\mathbf{y}$ under IGS transmission signaling are given by (16) and (17), respectively. Moreover, the covariance and pseudo-covariance matrices of the improper HWIs are given by (13) and (12), respectively. IGS provides additional degrees of freedom to appropriately design $\tilde{\mathbf{C}}_{\mathbf{x}}$ beside the transmit covariance matrix $\mathbf{C}_{\mathbf{x}}$, to improve the system performance.

\section{B. Hardware Impaired SIMO with Linear Combiner}

In this subsection, the instantaneous achievable rate expressions are derived for the ideal and non-ideal hardware in a conventional SIMO system with linear combiner. Furthermore, PGS and IGS transmission schemes are adopted in the nonideal hardware scenario.

1) Ideal Hardware Scenario: In the absence of hardware impairments, the received signal for ideal SIMO system is derived from (19) and is found to be $y_{\text {SIMO }}=\phi^{H} \mathbf{y}=$ $\phi^{\mathrm{H}} \mathbf{h} x+\phi^{\mathrm{H}} \mathbf{w}$ with $x \sim \mathcal{C N}\left(0, \sigma_{x}^{2}, 0\right)$. Hence the undesired signal $z$ is only comprised of PGS thermal noise with variance $\sigma_{z}^{2}=\phi^{\mathrm{H}} \mathbf{C}_{\mathbf{w}} \phi$ and the superposed RX signal $y_{\text {Simo }}$ has variance $\sigma_{y}^{2}=\left(\phi^{\mathrm{H}} \mathbf{h} \mathbf{h}^{H} \phi\right) \sigma_{x}^{2}+\sigma_{z}^{2}$. Based on Lemma 2, one can evaluate the differential entropy of $y$ and $z$ are given as $h(y)=\log _{2}\left((\pi e) \sigma_{y}^{2}\right)$ and $h(w)=\log _{2}\left((\pi e) \sigma_{w}^{2}\right)$, respectively. Therefore, using the achievable rate expression given by mutual information relation (21), we obtain

$$
R_{\text {SIMO - Ideal }}=B \log _{2}\left(1+\sigma_{x}^{2} \frac{\phi^{\mathrm{H}} \mathbf{h h}^{\mathrm{H}} \phi}{\phi^{\mathrm{H}} \mathbf{C}_{\mathbf{w}} \phi}\right) .
$$

Under ideal hardware assumption and perfect CSI at RX, optimal normalized receiver combiner $\phi=\frac{\mathbf{C}_{\mathbf{w}}{ }^{-1} \mathbf{h}}{\left\|\mathbf{C}_{\mathbf{w}}-\mathbf{h}\right\|_{2}}$ to maximize SNR and subsequently maximize achievable rate, is derived using Cauchy-Schwarz inequality.

2) Non-Ideal Hardware with PGS Transmission: Based on the non-ideal signal model in (19) and the PGS feature of $x$, the instantaneous achievable rate of HWI SIMO system is obtained using Lemma 2

$$
R_{\mathrm{SIMO}-\mathrm{PGS}}=\frac{B}{2} \log \left(\frac{\sigma_{y_{\mathrm{P}}}^{4}-\left|\tilde{\sigma}_{y_{\mathrm{P}}}^{2}\right|^{2}}{\sigma_{z}^{4}-\left|\tilde{\sigma}_{z}^{2}\right|^{2}}\right),
$$

where, the variance $\sigma_{y_{\mathrm{P}}}^{2}$ and pseudo-variance $\tilde{\sigma}_{y_{\mathrm{P}}}^{2}$ of the superposed signal $y_{\text {SIMO }}$ with proper Gaussian signal transmission are

$$
\begin{gathered}
\sigma_{y_{\mathrm{P}}}^{2}=\sigma_{x}^{2} \phi^{\mathrm{H}}\left(\overline{\mathbf{h}}_{1} \overline{\mathbf{h}}_{1}^{\mathrm{H}}+\overline{\mathbf{h}}_{2} \overline{\mathbf{h}}_{2}^{\mathrm{H}}\right) \phi+\phi^{\mathrm{H}} \mathbf{C}_{\mathbf{z}} \phi \\
\tilde{\sigma}_{y_{\mathrm{P}}}^{2}=\sigma_{x}^{2} \phi^{\mathrm{H}}\left(\overline{\mathbf{h}}_{1} \overline{\mathbf{h}}_{2}^{\mathrm{T}}+\overline{\mathbf{h}}_{2} \overline{\mathbf{h}}_{1}^{\mathrm{T}}\right) \phi^{*}+\phi^{\mathrm{H}} \tilde{\mathbf{C}}_{\mathbf{z}} \phi^{*} .
\end{gathered}
$$

SIMO receiver combining vector $\phi$ for PGS transmission is designed in (20). Moreover, the variance and pseudo-variance of superposed distortion signal $\phi^{\mathrm{H}} \hat{\mathbf{z}}$ are given by $\sigma_{z}^{2}=\phi^{\mathrm{H}} \mathbf{C}_{\mathbf{z}} \phi$ and $\sigma_{z}^{2}=\phi^{\mathrm{H}} \tilde{\mathbf{C}}_{\mathbf{z}} \phi^{*}$ respectively. Where,

$$
\begin{aligned}
& \mathbf{C}_{\mathbf{z}}=\boldsymbol{\Gamma}_{1}\left(\sigma_{\mathrm{T}}^{2} \mathbf{h h}^{\mathrm{H}}+\mathbf{C}_{\mathrm{R}}\right) \boldsymbol{\Gamma}_{1}^{\mathrm{H}}+\boldsymbol{\Gamma}_{2}\left(\sigma_{\mathrm{T}}^{2} \mathbf{h}^{*} \mathbf{h}^{\mathrm{T}}+\mathbf{C}_{\mathrm{R}}\right) \boldsymbol{\Gamma}_{2}^{\mathrm{H}} \\
& \tilde{\mathbf{C}}_{\mathbf{z}}=\boldsymbol{\Gamma}_{1}\left(\sigma_{\mathrm{T}}^{2} \mathbf{h h}^{\mathrm{H}}+\mathbf{C}_{\mathrm{R}}\right) \boldsymbol{\Gamma}_{2}^{\mathrm{T}}+\boldsymbol{\Gamma}_{2}\left(\sigma_{\mathrm{T}}^{2} \mathbf{h}^{*} \mathbf{h}^{\mathrm{T}}+\mathbf{C}_{\mathrm{R}}\right) \boldsymbol{\Gamma}_{1}^{\mathrm{T}},
\end{aligned}
$$

From (34), it is evident that the SIMO-LC achievable rate with PGS transmission can only be improved by improving the transmit variance $\sigma_{x}^{2}$ unlike IGS transmission as discussed in the following subsection. 
3) Non-Ideal Hardware with IGS Transmission: Following the same procedure as in non-ideal SIMO under PGS, we obtain the rate expression for HWI SIMO system under IGS transmission signaling,

$$
R_{\mathrm{SIMO}-\mathrm{IGS}}=\frac{B}{2} \log \left(\frac{\sigma_{y_{\mathrm{I}}}^{4}-\left|\tilde{\sigma}_{y_{\mathrm{I}}}^{2}\right|^{2}}{\sigma_{z}^{4}-\left|\tilde{\sigma}_{z}^{2}\right|^{2}}\right)
$$

where the variance and pseudo-variance of the received signal are given by

$$
\begin{gathered}
\sigma_{y_{\mathrm{I}}}^{2}=\phi^{\mathrm{H}}\left(\sigma_{x}^{2}\left(\overline{\mathbf{h}}_{1} \overline{\mathbf{h}}_{1}^{\mathrm{H}}+\overline{\mathbf{h}}_{2} \overline{\mathbf{h}}_{2}^{\mathrm{H}}\right)+\tilde{\sigma}_{x}^{2} \overline{\mathbf{h}}_{1} \overline{\mathbf{h}}_{2}^{\mathrm{H}}+\tilde{\sigma}_{x}^{* 2} \overline{\mathbf{h}}_{2} \overline{\mathbf{h}}_{1}^{\mathrm{H}}+\mathbf{C}_{\mathbf{z}}\right) \phi . \\
\tilde{\sigma}_{y_{\mathrm{I}}}^{2}=\phi^{\mathrm{H}}\left(\sigma_{x}^{2}\left(\overline{\mathbf{h}}_{1} \overline{\mathbf{h}}_{2}^{\mathrm{T}}+\overline{\mathbf{h}}_{2} \overline{\mathbf{h}}_{1}^{\mathrm{T}}\right)+\tilde{\sigma}_{x}^{2} \overline{\mathbf{h}}_{1} \overline{\mathbf{h}}_{\mathbf{1}}^{\mathrm{T}}+\tilde{\sigma}_{\mathbf{x}}^{* \mathbf{2}} \overline{\mathbf{h}}_{\mathbf{2}} \overline{\mathbf{h}}_{\mathbf{2}}^{\mathrm{T}}+\tilde{\mathbf{C}}_{\mathbf{z}}\right) \phi^{*} .
\end{gathered}
$$

SIMO receiver combining vector $\phi$ for IGS transmission is designed in (20). Moreover, the variance and pseudo-variance of superposed distortion signal $\phi^{\mathrm{H}} \hat{\mathbf{z}}$ are given by $\sigma_{z}^{2}=\phi^{\mathrm{H}} \mathbf{C}_{\mathbf{z}} \phi$ and $\tilde{\sigma}_{z}^{2}=\phi^{\mathrm{H}} \tilde{\mathbf{C}}_{\mathbf{z}} \phi^{*}$, respectively, where $\tilde{\mathbf{C}}_{\mathbf{z}}$ and $\tilde{\mathbf{C}}_{\mathbf{z}}$ are given in (37) and (38), respectively. We can jointly design variance and pseudo-variance to maximize achievable rate of HWI SIMO system under IGS transmit signaling, by solving the following optimization problem

$$
\begin{array}{ll}
\mathbf{P 1}: & \max _{\sigma_{x}^{2}, \tilde{\sigma}_{x}^{2}} \mathrm{R}_{\text {SIMO-IGS }}\left(\sigma_{x}^{2}, \tilde{\sigma}_{x}^{2}\right) \\
& \text { s. t. } \quad 0 \leqslant\left|\tilde{\sigma}_{x}^{2}\right| \leqslant \sigma_{x}^{2} \leqslant p .
\end{array}
$$

\section{HWI SIMO System with Selection Combiner}

Employing sub-optimal selection combining scheme offers less complex systems with high energy efficiency. We first express the achievable rate expression in case of ideal hardware and then we present the practical scenario of non-ideal hardware.

1) Ideal Hardware Scenario: Consider the ideal SIMO system under PGS using selection combining scheme, the received signal at the $j^{\text {th }} \mathrm{RX}$ branch is $y_{j}=h_{j} x+w_{j} \quad 1 \leqslant$ $j \leqslant N$. Based on Lemma 2, the differential entropy of $y$ and $w$ are derived as $h(y)=\log _{2}\left((\pi e) \sigma_{y}^{2}\right)$ and $h\left(w_{j}\right)=$ $\log _{2}\left((\pi e) \sigma_{w}^{2}\right)$, respectively. Therefore using (21), the instantaneous rate achieved by the selection combining scheme is

$$
R_{\text {SIMO-SC }- \text { Ideal }}=\max _{j} B \log _{2}\left(1+\frac{\left|h_{j}\right|^{2} \sigma_{x}^{2}}{\sigma_{w}^{2}}\right) .
$$

2) Non-Ideal Hardware with PGS Transmission: After adopting PGS for signal transmission, we obtain the following achievable rate expression $R_{\mathrm{SIMO}-\mathrm{PGS}}^{j}$ for the selection combining scheme with HWIs

$$
R_{\mathrm{SIMO}-\mathrm{SC}-\mathrm{PGS}}=\max _{j} \frac{B}{2} \log \left(\frac{\sigma_{y_{\mathrm{P} j}}^{4}-\left|\tilde{\sigma}_{y_{\mathrm{P} j}}^{2}\right|^{2}}{\sigma_{z_{j}}^{4}-\left|\tilde{\sigma}_{z_{j}}^{2}\right|^{2}}\right)
$$

where $\sigma_{y_{\mathrm{P} j}}^{2}$ and $\tilde{\sigma}_{y_{\mathrm{P} j}}^{2}$ of the received signal at the $j^{\text {th }}$ receiver branch are written, respectively, as

$$
\begin{gathered}
\sigma_{y_{\mathrm{P} j}}^{2}=\sigma_{x}^{2}\left(\left|\bar{h}_{1 j}\right|^{2}+\left|\bar{h}_{2 j}\right|^{2}\right)+\sigma_{z_{j}}^{2} \\
\tilde{\sigma}_{y_{\mathrm{P} j}}^{2}=2 \bar{h}_{1 j} \bar{h}_{2 j} \sigma_{x}^{2}+\tilde{\sigma}_{z_{j}}^{2} .
\end{gathered}
$$

In addition, $\sigma_{z_{j}}^{2}$ and $\tilde{\sigma}_{z_{j}}^{2}$ of the asymmetric aggregated distortion component observed at the $j^{\text {th }}$ receiver stream is given, respectively, as

$$
\begin{gathered}
\sigma_{z_{j}}^{2}=\left(\left|\Upsilon_{1}\right|^{2}+\left|\Upsilon_{2}\right|^{2}\right)\left(\sigma_{\mathrm{T}}^{2}\left|h_{j}\right|^{2}+\sigma_{\mathrm{R}}^{2}\right) \\
\tilde{\sigma}_{z_{j}}^{2}=\left(\Upsilon_{1}^{2}+\Upsilon_{2}^{2}\right)\left(\sigma_{\mathrm{T}}^{2}\left|h_{j}\right|^{2}+\sigma_{\mathrm{R}}^{2}\right) .
\end{gathered}
$$

3) Non-Ideal Hardware with IGS Transmission: The impact of IGS transmission on the achievable rate of HWI SIMO with selection combining is observed as

$$
R_{\mathrm{SIMO}-\mathrm{IGS}}=\max _{j} \frac{B}{2} \log \left(\frac{\sigma_{y_{\mathrm{I} j}}^{4}-\left|\tilde{\sigma}_{y_{\mathrm{I} j}}^{2}\right|^{2}}{\sigma_{z_{j}}^{4}-\left|\tilde{\sigma}_{z_{j}}^{2}\right|^{2}}\right),
$$

where $\sigma_{y_{\mathrm{I} j}}^{2}$ and $\tilde{\sigma}_{y_{\mathrm{I} j}}^{2}$ are expressed, respectively, as

$$
\begin{gathered}
\sigma_{y_{\mathrm{I} j}}^{2}=\sigma_{y_{\mathrm{P} j}}^{2}+\bar{h}_{1 j} \bar{h}_{2 j}^{*} \tilde{\sigma}_{x}^{2}+\bar{h}_{2 j} \bar{h}_{1 j}^{*} \tilde{\sigma}_{x}^{* 2} \\
\tilde{\sigma}_{y_{\mathrm{I} j}}^{2}=\tilde{\sigma}_{y_{\mathrm{P} j}}^{2}+\tilde{\sigma}_{x}^{2} \bar{h}_{1 j}^{2}+\tilde{\sigma}_{x}^{* 2} \bar{h}_{2 j}^{2} .
\end{gathered}
$$

The statistical characteristics of the transmitted signal can be optimized to reduce the distortion effect and increase the achievable rate using IGS by solving following optimization problem

$$
\begin{array}{ll}
\text { P2 : } & \max _{\sigma_{x}^{2}, \tilde{\sigma}_{x}^{2}} R_{\text {SIMO - IGS }}^{j}\left(\sigma_{x}^{2}, \tilde{\sigma}_{x}^{2}\right) \\
& \text { s. t. } \quad 0 \leqslant\left|\tilde{\sigma}_{x}^{2}\right| \leqslant \sigma_{x}^{2} \leqslant p .
\end{array}
$$

The transmit parameters are optimized to achieve maximum instantaneous achievable rate of SIMO-LC and SIMO-SC systems with IGS transmission.

\section{Achievable Rate Optimization of SimO Systems}

In this section, we design the transmitted signal, to maximize achievable rate in the presence of different HWIs in SIMO system under LC and SC diversity schemes. The main goal of the system design is to jointly optimize the statistical characteristics such as variance and pseudo-variance of the transmitted signal to maximize the achievable rate under IGS transmission. Subsequently, we propose an adaptive scheme to switch transmission between maximal IGS and PGS to maximize the end-to-end achievable rate.

\section{A. SIMO - Linear Combining Scheme}

Throughout the following optimization framework, we jointly optimize transmit variance and pseudo-variance in order to maximize the achievable rate. Meanwhile, maximizing $\mathrm{R}_{\text {SIMO-IGS }}$ is equivalent to maximizing $\Xi_{\text {SIMO-IGS }}$ thanks to the increasing nature of the logarithmic function, where

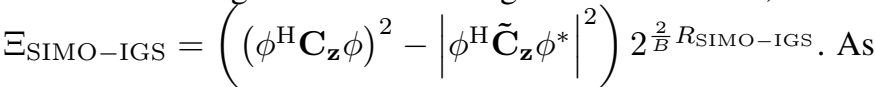
such, the optimization problem is written as,

$$
\begin{array}{ll}
\text { P3 : } & \max _{\sigma_{x}^{2}, \tilde{\sigma}_{x}^{2}} \Xi_{\text {SIMO - IGS }}\left(\sigma_{\mathrm{x}}^{2}, \tilde{\sigma}_{\mathrm{x}}^{2}\right) \\
& \text { s. t. } 0 \leqslant\left|\tilde{\sigma}_{x}^{2}\right| \leqslant \sigma_{x}^{2} \leqslant p,
\end{array}
$$

where $\Xi_{\mathrm{SIMO}-\text { IGS }}\left(\sigma_{\mathrm{x}}^{2}, \tilde{\sigma}_{\mathrm{x}}^{2}\right)=\sigma_{y_{\mathrm{I}}}^{4}-\left|\tilde{\sigma}_{y_{\mathrm{I}}}^{2}\right|^{2}$ and the variance $\sigma_{y_{\mathrm{I}}}^{2}$ and pseudo-variance $\tilde{\sigma}_{y_{\mathrm{I}}}^{2}$ of the received signal under IGS 
TABLE I: SIMO System Parameters

\begin{tabular}{|c|c|c|c|}
\hline \multicolumn{2}{|r|}{ SIMO-LC System Parameters } & \multicolumn{2}{|r|}{ SIMO-SC System Parameters } \\
\hline Symbols & $\begin{array}{c}\text { Value } \\
\end{array}$ & Symbols & $\begin{array}{c}\text { Value } \\
\end{array}$ \\
\hline$q_{11}$ & $4 \Re\left\{\phi^{\mathrm{H}} \overline{\mathbf{h}}_{1} \overline{\mathbf{h}}_{2}^{\mathrm{H}} \phi\right\}^{2}-\left|\phi^{\mathrm{H}} \overline{\mathbf{h}}_{1} \overline{\mathbf{h}}_{1}^{\mathrm{T}} \phi^{*}\right|^{2}-\left|\phi^{\mathrm{H}} \overline{\mathbf{h}}_{2} \overline{\mathbf{h}}_{2}^{\mathrm{T}} \phi^{*}\right|^{2}-2 \Re\left\{m_{4}\right\}$ & $e_{11}$ & $4 \Re\left\{\bar{h}_{1 j} \bar{h}_{2 j}^{*}\right\}^{2}-\left|\bar{h}_{1 j}^{2}\right|^{2}-\left|\bar{h}_{2 j}^{2}\right|^{2}-2 \Re\left\{d_{4}\right\}$ \\
\hline$q_{12}$ & $-4 \Re\left\{\phi^{\mathrm{H}} \overline{\mathbf{h}}_{1} \overline{\mathbf{h}}_{2}^{\mathrm{H}} \phi\right\} \Im\left\{\phi^{\mathrm{H}} \overline{\mathbf{h}}_{1} \overline{\mathbf{h}}_{2}^{\mathrm{H}} \phi\right\}+2 \Im\left\{m_{4}\right\}$ & $e_{12}$ & $-4 \Re\left\{\bar{h}_{1 j} \bar{h}_{2 j}^{*}\right\} \Im\left\{\bar{h}_{1 j} \bar{h}_{2 j}^{*}\right\}+2 \Im\left\{d_{4}\right\}$ \\
\hline$q_{13}$ & $2 n_{1} \Re\left\{\phi^{\mathrm{H}} \overline{\mathbf{h}}_{1} \overline{\mathbf{h}}_{2}^{\mathrm{H}} \phi\right\}-\Re\left\{m_{2}\right\}$ & $e_{13}$ & $2 b_{1} \Re\left\{\bar{h}_{1 j} \bar{h}_{2 j}^{*}\right\}-\Re\left\{d_{2}\right\}$ \\
\hline$q_{22}$ & $4 \Im\left\{\phi^{\mathrm{H}} \overline{\mathbf{h}}_{1} \overline{\mathbf{h}}_{2}^{\mathrm{H}} \phi\right\}^{2}-\left|\phi^{\mathrm{H}} \overline{\mathbf{h}}_{1} \overline{\mathbf{h}}_{1}^{\mathrm{T}} \phi^{*}\right|^{2}-\left|\phi^{\mathrm{H}} \overline{\mathbf{h}}_{2} \overline{\mathbf{h}}_{2}^{\mathrm{T}} \phi^{*}\right|^{2}+2 \Re\left\{m_{4}\right\}$ & $e_{22}$ & $4 \Im\left\{\bar{h}_{1 j} \bar{h}_{2 j}^{*}\right\}^{2}-\left|\bar{h}_{1 j}^{2}\right|^{2}-\left|\bar{h}_{2 j}^{2}\right|^{2}+2 \Re\left\{d_{4}\right\}$ \\
\hline$q_{23}$ & $-2 n_{1} \Im\left\{\phi^{\mathrm{H}} \overline{\mathbf{h}}_{1} \overline{\mathbf{h}}_{2}^{\mathrm{H}} \phi\right\}+\Im\left\{m_{2}\right\}$ & $e_{23}$ & $-2 b_{1} \Im\left\{h_{1 j} \bar{h}_{2 j}^{*}\right\}+\Im\left\{d_{2}\right\}$ \\
\hline$q_{33}$ & $n_{1}^{2}-\left|n_{2}\right|^{2}$ & $e_{33}$ & $-b_{1}^{2}-\left|b_{2}\right|^{2}$ \\
\hline$p_{1}$ & $4 \Re\left\{\phi^{\mathrm{H}} \overline{\mathbf{h}}_{1} \overline{\mathbf{h}}_{2}^{\mathrm{H}} \phi\right\}\left(\phi^{\mathrm{H}} \mathbf{C}_{\mathbf{z}} \phi\right)-2 \Re\left\{m_{3}\right\}$ & $f_{1}$ & $4 \Re\left\{\bar{h}_{1 j} \bar{h}_{2 j}^{*}\right\} \sigma_{z}^{2}-2 \Re\left\{d_{3}\right\}$ \\
\hline$p_{2}$ & $-4 \Im\left\{\phi^{\mathrm{H}} \overline{\mathbf{h}}_{1} \overline{\mathbf{h}}_{2}^{\mathrm{H}} \phi\right\}\left(\phi^{\mathrm{H}} \mathbf{C}_{\mathbf{z}} \phi\right)+2 \Im\left\{m_{3}\right\}$ & $f_{2}$ & $-4 \Im\left\{\bar{h}_{1 j} \bar{h}_{2 j}^{*}\right\} \sigma_{z}^{2}+2 \Im\left\{d_{3}\right\}$ \\
\hline$p_{3}$ & $2 n_{1}\left(\phi^{\mathrm{H}} \mathbf{C}_{\mathbf{z}} \phi\right)-m_{1}$ & $f_{3}$ & $2 b_{1} \sigma_{z}^{2}-d_{1}$ \\
\hline$r$ & $\left(\phi^{\mathrm{H}} \mathbf{C}_{\mathbf{z}} \phi\right)^{2}-\left|\phi^{\mathrm{H}} \tilde{\mathbf{C}}_{\mathbf{z}} \phi^{*}\right|^{2}$ & $g$ & $\sigma_{z}^{4}-\left|\tilde{\sigma}_{z}^{2}\right|^{2}$ \\
\hline$m_{1}$ & $n_{2}\left(\phi \tilde{\mathbf{C}}_{z}^{*} \phi^{T}\right)+n_{2}^{*}\left(\phi^{\mathrm{H}} \tilde{\mathbf{C}}_{\mathbf{z}} \phi^{*}\right)$ & $d_{1}$ & $b_{2} \tilde{\sigma}_{z}^{2 *}+b_{2}^{*} \tilde{\sigma}_{z}^{2}$ \\
\hline$m_{2}$ & $n_{2}\left(\phi \overrightarrow{\mathbf{h}}_{1}^{H} \overline{\mathbf{h}}_{\mathbf{1}}^{*} \phi^{T}\right)+n_{2}^{*}\left(\phi^{\mathrm{H}} \overline{\mathbf{h}}_{2} \overline{\mathbf{h}}_{2}^{\mathrm{T}} \phi^{*}\right)$ & $d_{2}$ & $b_{2} \bar{h}_{2 j}^{2 *}+b_{2}^{*} \bar{h}_{1 j}^{2}$ \\
\hline$m_{3}$ & $\left(\phi^{\mathrm{H}} \overline{\mathbf{h}}_{1} \overline{\mathbf{h}}_{1}^{\mathrm{T}} \phi^{*}\right)\left(\phi \tilde{\mathbf{C}}_{z}^{*} \phi^{T}\right)+\left(\phi \overline{\mathbf{h}}_{2}^{H} \overline{\mathbf{h}}_{2}^{*} \phi^{T}\right)\left(\phi^{\mathrm{H}} \tilde{\mathbf{C}}_{\mathbf{z}} \phi^{*}\right)$ & $d_{3}$ & $\bar{h}_{1 j}^{2} \tilde{\sigma}_{z}^{2 *}+\bar{h}_{2 j}^{2 *} \tilde{\sigma}_{z}^{2}$ \\
\hline$m_{4}$ & $\left(\phi^{\mathrm{H}} \overline{\mathbf{h}}_{1} \overline{\mathbf{h}}_{1}^{\mathrm{T}} \phi^{*}\right)\left(\phi \overline{\mathbf{h}}_{2}^{H} \overline{\mathbf{h}}_{2}^{*} \phi^{T}\right)$ & $d_{4}$ & $\bar{h}_{1 j}^{2} \bar{h}_{2 j}^{2 *}$ \\
\hline$n_{1}$ & $\begin{array}{l}\phi^{\mathrm{H}}\left(\overline{\mathbf{h}}_{1} \overline{\mathbf{h}}_{1}^{\mathrm{H}}+\overline{\mathbf{h}}_{2} \overline{\mathbf{h}}_{2}^{\mathrm{H}}\right) \phi \\
\phi^{\mathrm{H}}\left(\overline{\mathbf{h}}_{1} \overline{\mathbf{h}}_{\mathrm{T}}^{\mathrm{T}}+\overline{\mathbf{h}}_{2} \overline{\mathbf{h}}^{\mathrm{T}}\right) \phi^{*}\end{array}$ & $b_{1}$ & $\left|\bar{h}_{1 j}\right|^{2}+\left|\bar{h}_{2 j}\right|^{2}$ \\
\hline$n_{2}$ & $\phi^{\mathrm{n}}\left(\mathbf{h}_{1} \mathbf{h}_{2}^{1}+\mathbf{h}_{2} \mathbf{h}_{1}^{1}\right) \phi^{*}$ & $b_{2}$ & \\
\hline
\end{tabular}

transmission are given by (40) and (41) respectively. To solve P3, we present all complex quantities as the superposition of their real and imaginary parts. Thus, the objective function can be equivalently expressed in its real form as

$$
\begin{aligned}
\Xi_{\text {SIMO-IGS }}= & q_{11} \Re\left\{\tilde{\sigma}_{x}^{2}\right\}^{2}+q_{22} \Im\left\{\tilde{\sigma}_{x}^{2}\right\}^{2}+q_{33} \sigma_{x}^{4}+p_{3} \sigma_{x}^{2}+r \\
& +2 q_{12} \Re\left\{\tilde{\sigma}_{x}^{2}\right\} \Im\left\{\tilde{\sigma}_{x}^{2}\right\}+2 q_{13} \sigma_{x}^{2} \Re\left\{\tilde{\sigma}_{x}^{2}\right\} \\
& +2 q_{23} \sigma_{x}^{2} \Im\left\{\tilde{\sigma}_{x}^{2}\right\}+p_{1} \Re\left\{\tilde{\sigma}_{x}^{2}\right\}+p_{2} \Im\left\{\tilde{\sigma}_{x}^{2}\right\}
\end{aligned}
$$

where, the SIMO-LC system parameters are presented in Table I. Moreover, the optimization problem $\mathbf{P 3}$ can be equivalently presented in a vector form. First, we write the vector $\mathbf{s}$ that captures the transmit variance, and real and imaginary parts of pseudo-variance as $\mathbf{s}=\left[\Re\left\{\tilde{\sigma}_{x}^{2}\right\} \Im\left\{\tilde{\sigma}_{x}^{2}\right\} \sigma_{x}^{2}\right]^{T}$ for SIMO-LC under IGS transmission. Then, we express the equivalent problem as a quadratic-constraint quadratic-programming (QCQP) with constraints $\mathrm{C} 1$ and $\mathrm{C} 2$ as

$$
\begin{array}{r}
\mathbf{P 4}: \max _{\mathbf{s}} \mathbf{s}^{\mathrm{T}} \mathbf{Q} \mathbf{s}+\mathbf{s}^{\mathrm{T}} \mathbf{p}+r \\
\text { s.t. } \mathrm{C} 1: \mathbf{A}_{1} \mathbf{s} \leqslant \mathbf{b}, \\
\mathrm{C} 2: \mathbf{s}^{\mathrm{T}} \mathbf{A}_{2} \mathbf{s} \leqslant \mathbf{0},
\end{array}
$$

where $\mathbf{A}_{1}=\operatorname{diag}\left[\begin{array}{lll}0 & 0 & 1\end{array}\right]^{\mathrm{T}}, \mathbf{b}=\left[\begin{array}{lll}0 & 0 & \mathrm{P}_{\mathrm{T}}\end{array}\right]^{\mathrm{T}}$ and $\mathbf{A}_{2}=$ $\operatorname{diag}\left[\begin{array}{ll}1 & 1\end{array}-1\right]^{\mathrm{T}}$. As for the elements of symmetric matrix $\mathbf{Q} \in \mathbb{C}^{3 \times 3}$ and vector $\mathbf{p} \in \mathbb{C}^{3 \times 1}$, one can evaluate them using (51). This problem can be efficiently solved using interiorpoint method owing to the convex quadratic constraints.

\section{B. SIMO - Selection Combining Scheme}

Similarly, given the increasing nature of the logarithmic function, maximizing $R_{\text {SIMO - IGS }}^{j}$ in $\mathbf{P 2}$ is equivalent to maxi-

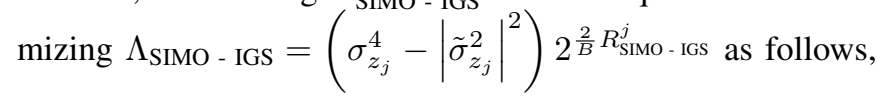

P5 : $\max _{\sigma_{x}^{2}, \tilde{\sigma}_{x}^{2}} \Lambda_{\text {SIMO - IGS }}\left(\sigma_{x}^{2}, \tilde{\sigma}_{x}^{2}\right)$

s. t. $0 \leqslant\left|\tilde{\sigma}_{x}^{2}\right| \leqslant \sigma_{x}^{2} \leqslant p$, where the objective function is $\Lambda_{\mathrm{SIMO} \text { - IGS }}=\sigma_{y_{\mathrm{I} j}}^{4}-\left|\tilde{\sigma}_{y_{\mathrm{I} j}}^{2}\right|^{2}$, with parameters as given in (49) and (50). To solve this optimization problem in real plane, we express each complex entity as summation of its real and imaginary part. Given $\Lambda_{\text {SIMO - IGS }}: \mathbb{C} \rightarrow \mathbb{R}, \Lambda_{\text {SIMO - IGS }}$ can be equivalently expressed as

$$
\begin{aligned}
\Lambda_{\text {SIMO - IGS }}= & e_{11} \Re\left\{\tilde{\sigma}_{x}^{2}\right\}^{2}+e_{22} \Im\left\{\tilde{\sigma}_{x}^{2}\right\}^{2}+2 e_{12} \Re\left\{\tilde{\sigma}_{x}^{2}\right\} \Im\left\{\tilde{\sigma}_{x}^{2}\right\} \\
& +e_{33} \sigma_{x}^{4}+2 e_{13} \sigma_{x}^{2} \Re\left\{\tilde{\sigma}_{x}^{2}\right\}+2 e_{23} \sigma_{x}^{2} \Im\left\{\tilde{\sigma}_{x}^{2}\right\} \\
& +f_{1} \Re\left\{\tilde{\sigma}_{x}^{2}\right\}+f_{2} \Im\left\{\tilde{\sigma}_{x}^{2}\right\}+f_{3} \sigma_{x}^{2}+g
\end{aligned}
$$

where, the SIMO-SC system parameters are presented in Table I. Moreover, the optimization problem P5 can be equivalently presented in a vector form. First, we write the vector $\mathbf{z}$ that captures the transmit variance, real and imaginary parts of pseudo-variance as $\mathbf{z}=\left[\Re\left\{\tilde{\sigma}_{x}^{2}\right\} \Im\left\{\tilde{\sigma}_{x}^{2}\right\} \sigma_{x}^{2}\right]^{T}$. Then we express the equivalent problem as a QCQP with convex constraints $\mathrm{C} 1$ and $\mathrm{C} 2$ as

$$
\begin{array}{r}
\mathbf{P 6}: \max _{\mathbf{z}} \mathbf{z}^{\mathrm{T}} \mathbf{E} \mathbf{z}+\mathbf{z}^{\mathrm{T}} \mathbf{f}+g \\
\text { s. t. } \mathrm{C} 1: \mathbf{A}_{1} \mathbf{z} \leqslant \mathbf{b}, \\
\mathrm{C} 2: \mathbf{z}^{\mathrm{T}} \mathbf{A}_{2} \mathbf{z} \leqslant \mathbf{0},
\end{array}
$$

where $\mathbf{E} \in \mathbb{C}^{3 \times 3}$ and $\mathbf{f} \in \mathbb{C}^{3 \times 1}$ can be evaluated using (52). The quadratic problem $\mathbf{P 6}$ can also be efficiently solved using interior point method.

\section{PGS - MIGS Adaptive Scheme}

One way to reduce the system complexity and optimization overhead is to switch the operation between PGS and maximally IGS (MIGS) according to the CSI and system parameters that are assumed to be known at the transmitter side. In the adaptive scheme, we derive the following switching criteria that maximize the system achievable rate: 
1) Transmit Power Criterion: The MIGS is assumed to have $\left|\tilde{\sigma}_{x}^{2}\right|=\sigma_{x}^{2}=p$, while the PGS system has $\sigma_{x}^{2}=p$ and $\left|\tilde{\sigma}_{x}^{2}\right|=0$. The MIGS outperforms its counterpart the PGS system in terms of achievable rate if $R_{\mathrm{MIGS}} \geqslant R_{\mathrm{PGS}}$. The rate condition is mapped to the following power condition after using the appropriate values for $\sigma_{x}^{2}$ and $\left|\tilde{\sigma}_{x}^{2}\right|$ :

$$
p \leq \frac{\left(2 \nu_{k} \sigma_{z k}^{2}-2 \Re\left\{\mu_{k}^{*} \tilde{\sigma}_{z k}^{2}\right\}\right)}{\left|\mu_{k}\right|^{2}-\nu_{k}^{2}-2 \nu_{k} v_{k}+2 \Re\left\{\mu_{k}^{*} \tau_{k}\right\}},
$$

where the coefficients with subscripts $k \in\{1,2\}$ for SIMOLC and SIMO-SC, respectively, are as follows:

- First, for SIMO-LC system with HWI, we deduce the following coefficients, $\nu_{1}=\phi^{\mathrm{H}}\left(\overline{\mathbf{h}}_{1} \overline{\mathbf{h}}_{2}^{\mathrm{H}}+\overline{\mathbf{h}}_{2} \overline{\mathbf{h}}_{1}^{\mathrm{H}}\right) \phi, \mu_{1}=$ $\phi^{\mathrm{H}}\left(\overline{\mathbf{h}}_{1} \overline{\mathbf{h}}_{1}^{\mathrm{T}}+\overline{\mathbf{h}}_{2} \overline{\mathbf{h}}_{\mathbf{2}}^{\mathrm{T}}\right) \phi^{*}, v_{1}=\phi^{\mathrm{H}}\left(\overline{\mathbf{h}}_{1} \overline{\mathbf{h}}_{1}^{\mathrm{H}}+\overline{\mathbf{h}}_{2} \overline{\mathbf{h}}_{2}^{\mathrm{H}}\right) \phi$ and $\tau_{1}=\phi^{\mathrm{H}}\left(\overline{\mathbf{h}}_{1} \overline{\mathbf{h}}_{2}^{\mathrm{T}}+\overline{\mathbf{h}}_{2} \overline{\mathbf{h}}_{1}^{\mathrm{T}}\right) \phi^{*}$. Moreover, $\sigma_{z_{1}}^{2}=\phi^{\mathrm{H}} \mathbf{C}_{\mathbf{z}} \phi$ and $\tilde{\sigma}_{z_{1}}^{2}=\phi^{\mathrm{H}} \tilde{\mathbf{C}}_{\mathbf{z}} \phi^{*}$ are given by (37) and (38) respectively.

- Next for SIMO-SC system with HWI, we obtain the coefficients $\nu_{2}=\bar{h}_{1 j} \bar{h}_{2 j}^{*}+\bar{h}_{2 j} \bar{h}_{1 j}^{*}, \mu_{2}=\bar{h}_{1 j}^{2}+\bar{h}_{2 j}^{2}$, $v_{2}=\left|\bar{h}_{1 j}\right|^{2}+\left|\bar{h}_{2 j}\right|^{2}$ and $\tau_{2}=2 \bar{h}_{1 j} \bar{h}_{2 j}$. Furthermore, the noise variance $\sigma_{z_{j}}^{2}$ and pseudo-variance $\tilde{\sigma}_{z_{j}}^{2}$ are given in (46) and (47) respectively.

2) Distortion Noise Criterion: Distortion noise level relative to the transmit signal level also imposes constraints on the employment of MIGS in comparison to PGS to maximize the achievable rate; $R_{\mathrm{MIGS}} \geqslant R_{\mathrm{PGS}}$. This leads to the following condition

$$
\begin{aligned}
\left(\operatorname{SDNR}_{\mathrm{IGS}}^{k}+1\right)^{2}-\left(\operatorname{SDNR}_{\mathrm{PGS}}^{k}+1\right)^{2} \geq \ldots \\
\frac{\left|\mu_{k}\right|^{2}+2 \Re\left\{\mu_{k}^{*}\left(\tau_{k}+\sigma_{x}^{-2} \tilde{\sigma}_{z k}^{2}\right)\right\}}{\sigma_{z k}^{4}}
\end{aligned}
$$

where, the coefficients $\nu_{k}, \mu_{k}, v_{k}$ and $\tau_{k}$ are already discussed in Section IV-C1 with $k=1,2$ for SIMO-LC and SIMO-SC respectively. Moreover, signal-to-distortion plus noise ratios for the MIGS and PGS are given as

$$
\mathrm{SDNR}_{\mathrm{MIGS}}^{k}=\frac{\sigma_{x}^{2}\left(v_{k}+\nu_{k}\right)}{\sigma_{z_{k}}^{2}} \text { and } \mathrm{SDNR}_{\mathrm{PGS}}^{k}=\frac{\sigma_{x}^{2} v_{k}}{\sigma_{z_{k}}^{2}} .
$$

Therefore, the MIGS scheme is advantageous over PGS scheme in terms of the achievable rate for the HWI SIMO-LC and SIMO-SC systems when the SDNR condition in (54) is satisfied.

\section{NumERICAL RESUltS}

In this section, we study the performance of multiple antenna systems subjected to HWIs and the proposed mitigation schemes. Through the numerical study, we consider MIMO system as well as two special cases of SIMO-LC and SIMO$\mathrm{SC}$ and investigate the system performance in terms of the average achievable rate. Furthermore, we examine the benefits reaped by adopting IGS in mitigating the HWIs compared to the PGS scheme. Moreover, we compare performance of the proposed adaptive scheme with the optimal IGS scheme for HWI-SIMO systems. However, the optimal solution of problem P1 is not easily tractable therefore we employ the

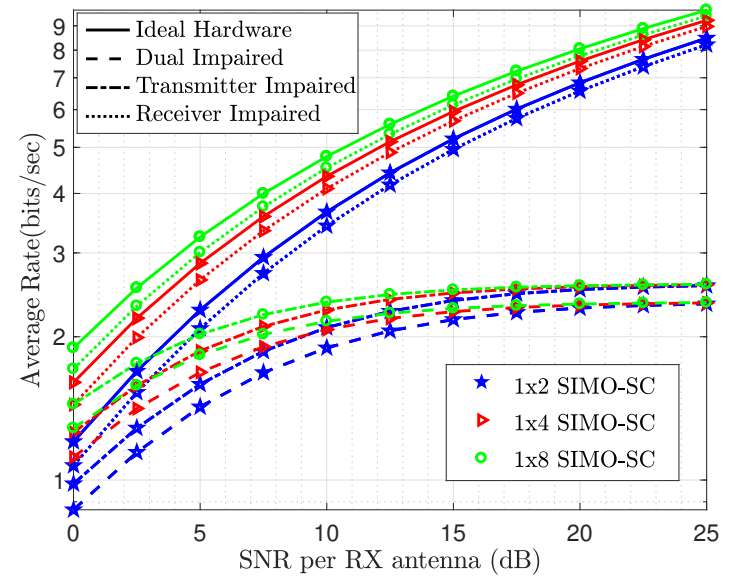

Fig. 4: SIMO average achievable rate vs. SNR for various impairment configurations

proposed MIGS scheme and study its performance relative to PGS scheme for HWI-MIMO systems.

As for the system parameters, we assume, unless otherwise specified, $p=1 \mathrm{~W}, B=1 \mathrm{~Hz}, \mathbf{C}_{\mathrm{T}}=\sigma_{\mathrm{T}}^{2} \mathbf{I}_{N_{\mathrm{T}}}$ and $\mathbf{C}_{\mathrm{R}}=$ $\sigma_{\mathrm{R}}^{2} \mathbf{I}_{N_{\mathrm{R}}}$ for simplicity. Moreover, we presume $\mathbf{A}_{\mathrm{T}}=a_{\mathrm{T}} \mathbf{I}_{N_{\mathrm{T}}}$, $\mathbf{A}_{\mathrm{R}}=a_{\mathrm{R}} \mathbf{I}_{N_{\mathrm{R}}}, \boldsymbol{\Theta}_{\mathrm{T}}=\theta_{\mathrm{T}} \mathbf{I}_{N_{\mathrm{T}}}$, and $\boldsymbol{\Theta}_{\mathrm{R}}=\theta_{\mathrm{R}} \mathbf{I}_{N_{\mathrm{R}}}$. It is worth to clarify that assuming identical $\mathrm{I} / \mathrm{Q}$ imbalance at all transceiver streams is justified by the employment of common oscillator for modulation and demodulation to the same carrier frequency in a spatially diverse system. In the rest of this section, the achievable rate performance is investigated against SNR, I/Q amplitude and phase imbalance, distortion levels and transceiver branches for non-ideal hardware with PGS, optimal IGS and maximal IGS transmission schemes.

\section{A. Performance Degradation}

Firstly, we quantify the performance degradation caused by HWIs in SIMO-SC configuration with varying receiver streams. Fig. 4 demonstrates the achievable rate deterioration caused by the dual impaired system, transmitter only impaired system and receiver only impaired system versus SNR per receiver antenna ranging from $0 \mathrm{~dB}$ to $25 \mathrm{~dB}$. We assumed the IRR $\Upsilon_{\mathrm{t}}=\Upsilon_{\mathrm{rk}}=20.3 \mathrm{~dB}$ dictated by $a_{\mathrm{T}}=a_{\mathrm{R}}=0.83$ and $\theta_{\mathrm{T}}=\theta_{\mathrm{R}}=3^{0}$ for all transceiver streams. Moreover, we suppose transmitter distortion $\sigma_{\mathrm{T}}^{2}=0.2$, receiver distortion plus thermal noise $\sigma_{\mathrm{R}}^{2}=1$ and varying number of receiver streams $N_{\mathrm{R}}=2,4 \& 8$. It is evident from Fig. 4 that the performance degradation caused by the dual impairments and transmitter only impairment is far more worse than the receiver only impairment despite of the multiple participating impaired receiving streams and single impaired transmitting branch. Moreover, the rate improvement with increasing SNR undergoes saturation in the presence of transmitter distortions, owing to the amplification of transmitter distortions along with the information signal. Interestingly, this results in the enhanced performance gap between ideal and nonideal hardware systems at higher SNR levels. Additionally, the rate improvement with increasing $N_{\mathrm{R}}$ becomes insignificant 


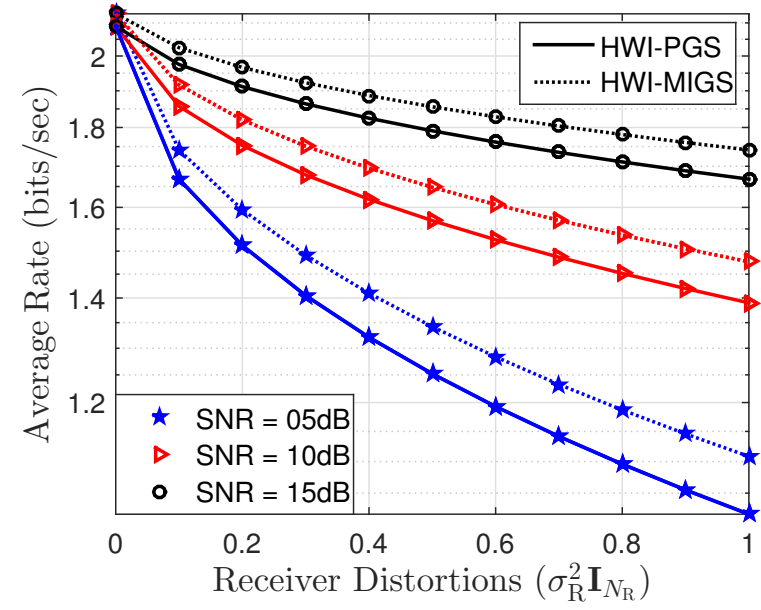

Fig. 5: MIMO average achievable rate vs. receiver distortions

rendering negligible diversity gain in the presence of HWIs. This motivates the study to adopt the appropriate compensation scheme to effectively mitigate the harmful effects of the HWIs.

\section{B. Effect of Distortion Noise Level}

Secondly, the impact of transmitter and receiver distortion levels is studied on HWI-MIMO system for varying SNR levels. Fig. 5 represents the average achievable rate performance of 5x5 MIMO system with IRR $\Upsilon_{\mathrm{tk}}=\Upsilon_{\mathrm{rk}}=19.77 \mathrm{~dB}$ dictated by $a_{\mathrm{T}}=a_{\mathrm{R}}=0.83$ and $\theta_{\mathrm{T}}=\theta_{\mathrm{R}}=5^{0}$ for all transceiver streams. We assume $\sigma_{\mathrm{T}}^{2}=0.5$ and analyze the system performance for $\sigma_{\mathrm{R}}^{2}=0-1$ with three SNR levels including $5 \mathrm{~dB}, 10 \mathrm{~dB}$, and $15 \mathrm{~dB}$. Evidently, the average achievable rate performance severely degrades with increasing receiver distortion levels. The two proposed transmission schemes; maximal IGS transmission with $\mathbf{C}_{\mathrm{x}}=\tilde{\mathbf{C}}_{\mathrm{x}}=\frac{P}{N_{\mathrm{T}}} \mathbf{I}_{N_{\mathrm{T}}}$ and PGS transmission with $\mathbf{C}_{\mathrm{x}}=\frac{P}{N_{\mathrm{T}}} \mathbf{I}_{N_{\mathrm{T}}}$ and $\tilde{\mathbf{C}}_{\mathrm{x}}=\mathbf{0}$ are investigated. Clearly, the drastic impact of HWIs can be effectively improved by employing MIGS for all presented receiver distortion and SNR levels. Moreover, the gain reaped by employing MIGS over traditional PGS is significantly high at higher impairment levels.

After that we study the degradation effect of transmitter distortions on a HWI-MIMO system with identical receiver distortions $\sigma_{\mathrm{R}}^{2}=1$ and 20dB IRR at all transmitter and receiver I/Q imbalanced streams. Owing to the immense degradation caused by the transmitter distortions, we employ $N_{\mathrm{T}}=10$ transmitter and $N_{\mathrm{R}}=15$ receiver streams to maintain reasonable system performance within limited power budget. We analyze the average achievable rate of the adopted MIMO system for varying transmitter distortions $\sigma_{\mathrm{T}}^{2}=0-1$ with three different SNR levels as shown in Fig. 6. Intuitively, the degradation caused by transmitter distortions is far worse than that of receiver distortions given same I/Q imbalance. Moreover, the performance gain obtained by increasing SNR undergoes saturation for higher distortion levels. It is merely due to the fact that increasing transmit power also amplifies transmitter distortions. Clearly, MIGS outperforms PGS trans-

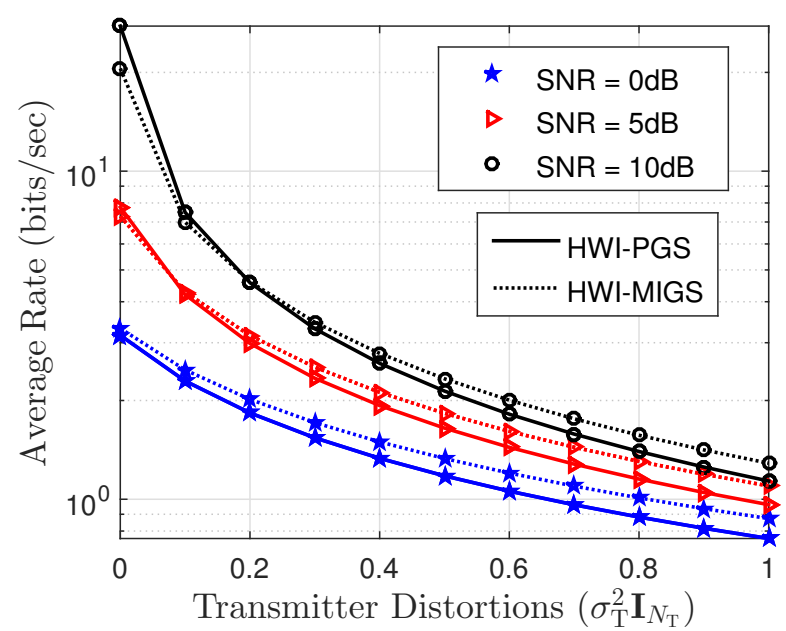

Fig. 6: MIMO average achievable rate vs. transmitter distortions

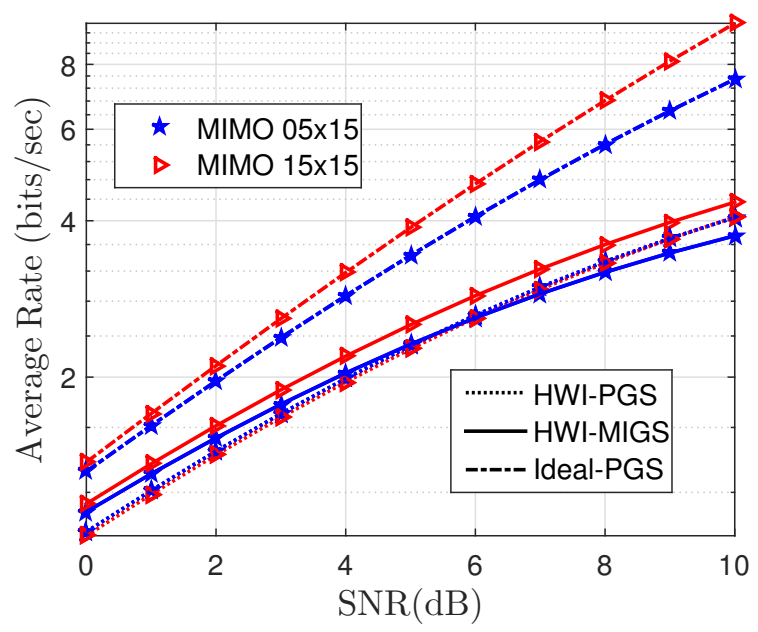

Fig. 7: MIMO average achievable rate vs. SNR

mission for lower SNR and higher impairment levels. whereas PGS is the preferred choice for higher SNR with negligible transmitter distortions.

\section{Effect of SNR on Achievable Rates}

Next, the average achievable rate performance of HWIMIMO and HWI-SIMO systems is evaluated for a range of SNR levels with varying number of transceiver systems. Fig. 7 represents the average achievable rate of $05 \times 15$ and $15 \times 15$ MIMO systems versus SNR ranging from 0dB-10dB for the ideal system with PGS transmission as well as HWI system employing PGS and MIGS transmission. Evidently, HWI-MIMO systems average rate performance is inferior to that of ideal-MIMO system. In addition, the performance gain of HWI-MIMO system obtained by increasing SNR and transceiver streams is also insignificant relative to that of ideal-MIMO system. Increased transmitter diversity is assumed to improve the system performance as depicted by Ideal-PGS, however the achievable rate performance of HWIPGS does not improve with increasing transmitter streams. 


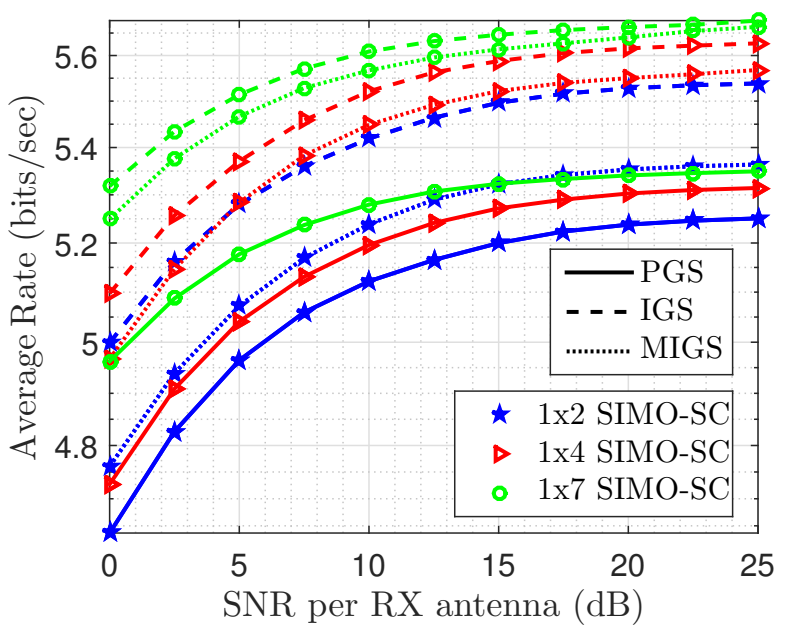

Fig. 8: SIMO-SC average achievable rate vs. SNR for various $N_{\mathrm{R}}$

It is merely because of the fact that increasing $N_{\mathrm{T}}$ also increases transmitter impairments and thus it fails to improve any system performance. In addition, careful analysis depicts that increasing SNR amplifies the transmitter HWIs rendering it ineffective to improve system throughput. On the other hand, IGS transmission for HWI-MIMO can enable higher system throughput with increasing $N_{\mathrm{T}}$ by effectively mitigating the HWIs. Interestingly, MIGS outperforms PGS for higher transceiver streams and lower SNR conditions. However, this gain diminishes with increasing SNR and then the adaptive switching scheme from MIGS to PGS can be employed to maintain system performance. For example, the adaptive transmission scheme can be switched from MIGS to PGS beyond 6dB SNR for the adopted $05 \times 15$ MIMO system to attain superior system performance. Whereas, MIGS outperforms PGS for 15x15 MIMO system in the given SNR range. In conclusion, adopting IGS can enable improving the performance of MIMO systems with HWI by increasing $N_{\mathrm{T}}$, which could not be achieved with the traditional signaling scheme, i.e., PGS.

The averaged achievable rate is also analyzed for hardware impaired SIMO-SC system with SNR ranging from $0 \mathrm{~dB}$ to $25 \mathrm{~dB}$ for varying number of receiver branches $N_{\mathrm{R}}=2,4 \& 7$. We assume $a_{\mathrm{T}}=a_{\mathrm{R}}=0.6$ and $\theta_{\mathrm{T}}=\theta_{\mathrm{R}}=3^{0}$ in this example. According to Fig. 8, increasing system diversity $N_{\mathrm{R}}$ and boosting SNR, both offer improved data-rates. However, the gain reaped by boosting SNR is more prominent for fewer $N_{\mathrm{R}}$ but undergoes saturation for higher order $N_{\mathrm{R}}$. Therefore, we can safely conclude that the presence of HWI imposes a huge challenge on multi-antenna systems to attain higher order data-rates. In addition, careful analysis depict that the deteriorating effect of HWIs is considerably mitigated in the presence of IGS and MIGS as compared to the counterpart PGS. Interestingly, MIGS performance becomes close to optimized IGS for large number of receiver streams eliminating the optimization overhead.

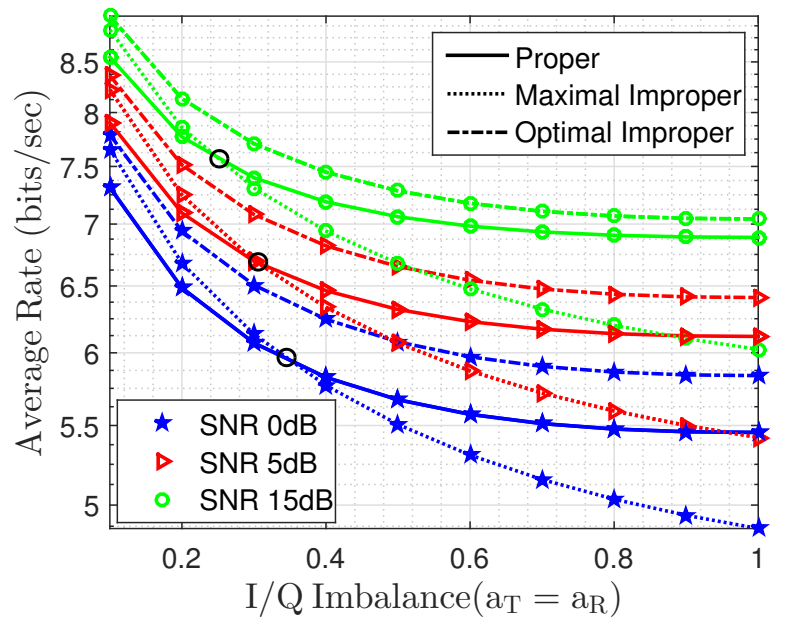

Fig. 9: SIMO-LC average achievable rate vs. $a_{\mathrm{T}}=a_{\mathrm{R}}$ for various SNR

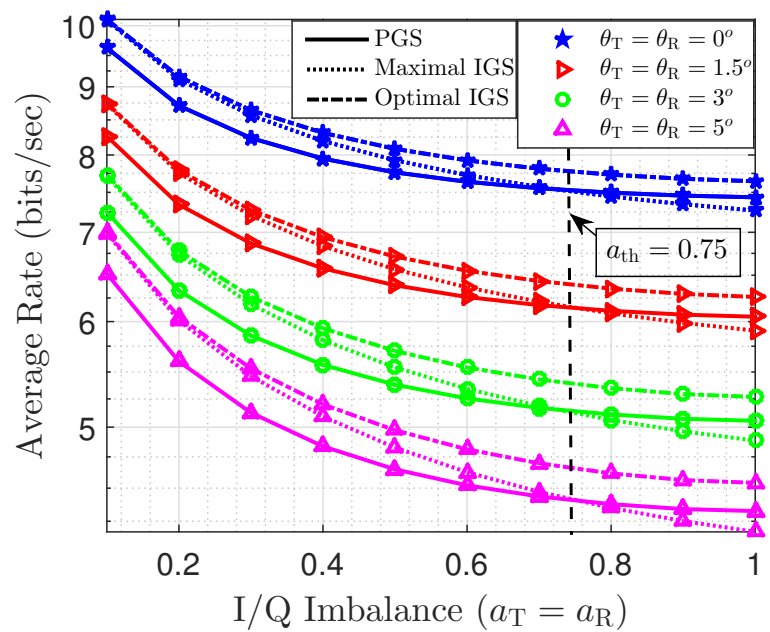

Fig. 10: SIMO-SC average achievable rate vs. I/Q imbalances

\section{Effect of $I / Q$ imbalance Level}

Later, the average achievable rate is analyzed for the HWI SIMO-LC system for the three different transmission schemes. System performance has been analyzed over a range of I/Q amplitude errors $a_{\mathrm{T}}=a_{\mathrm{R}}$ for a given fixed rotational translation of $\theta_{\mathrm{T}}=\theta_{\mathrm{R}}=1^{0}$. The achievable rate is studied for varying levels of SNR assuming $N_{\mathrm{R}}=3$ and $\sigma_{\mathrm{T}}^{2}=\sigma_{\mathrm{R}}^{2}=0.6$ as shown in Fig. 9. Evidently, the system performance decreases drastically with increasing I/Q amplitude error. However, this degradation can be dampened by increasing SNR. Moreover, optimized IGS scheme performs way better than the MIGS and PGS schemes by efficiently mitigating HWI especially in low SNR regime. It is important to highlight that the low-complexity adaptive scheme can be employed to switch between MIGS and PGS depending on the estimated amplitude error and SNR as encircled in Fig. 9. Generally, MIGS outperforms PGS for low $a_{\mathrm{T}}$ and $a_{\mathrm{R}}$, whereas PGS is preferred choice for medium to high $a_{\mathrm{T}}$ and $a_{\mathrm{R}}$ levels, in case of adaptive scheme. The switching takes place according to the thresholds discussed in Section IV-C. 
Then, the averaged achievable rate is analyzed for the SIMO-SC scheme at various I/Q amplitude and phase translation error values. The simulation results are obtained for $\mathrm{N}_{\mathrm{R}}=2, \mathrm{SNR}=30 \mathrm{~dB}, a_{\mathrm{T}}=a_{\mathrm{R}}$ ranging from $0-1$ and $\theta_{\mathrm{T}}=\theta_{\mathrm{R}}=0^{0}, 1.5^{0}, 3^{0}, 5^{0}$ as shown in Fig. 10. Clearly average achievable rate decreases with the increase in amplitude error. However, achievable rate rapidly decreases with increasing rotational error e.g. at $\theta_{\mathrm{T}}=\theta_{\mathrm{R}}=5^{0}$. Furthermore, at maximal amplitude error $a_{\mathrm{T}}=a_{\mathrm{R}}=1$, phase translation of $5^{0}$ renders around $50 \%$ decrease in achievable rate relative to the $0^{0}$ phase error. Subsequently, the deteriorating effect of HWI is considerably mitigated in the presence of optimal IGS as compared to its counterpart PGS, especially for high phase error values. In this illustration, MIGS outperforms PGS till $a_{\mathrm{th}}=0.75$ however PGS offers improved achievable rate performance for $a_{\mathrm{T}}=a_{\mathrm{R}} \geqslant 0.75$, under the proposed adaptive scheme. For small to medium amplitude errors, MIGS performs as good as optimized IGS, eliminating fine-tuning requirement and reducing system complexity. Interestingly, phase rotational error does not impose any condition on the adaptive scheme switching threshold.

\section{E. Effect of Receiver Branches}

Eventually, we investigate the effect of increasing $N_{R}$ from 1 to 6 for varying distortion levels due to the non-linear transfer of various aforementioned transceiver blocks. For simulation, we assume $\sigma_{\mathrm{T}}^{2}=\sigma_{\mathrm{R}}^{2}=0.5,0.75 \& 1, a_{\mathrm{T}}=a_{\mathrm{R}}=1$ , $\theta_{\mathrm{T}}=\theta_{\mathrm{R}}=3^{0}$. Fig. 11 depicts the degradation caused by the varying level of additive transceiver distortions given SNR $=25 \mathrm{~dB}$. Evidently, increasing additive distortions significantly deteriorate system performance. Whereas, increasing number of receiver branches counters HWI and struggles to improve the overall achievable rate while employing SC receiver combining scheme. Verily, optimized IGS outperforms PGS and MIGS in all presented scenarios. Intriguingly, MIGS can be adopted to improve system performance relative to the PGS transmission $N_{\mathrm{R}_{\mathrm{th}}} \geqslant 2$ under the adopted parameters. Moreover, MIGS performs equally good for higher order $N_{\mathrm{R}}$ as presented in Fig. 11, eliminating the need for joint optimization while efficiently mitigating various HWIs.

The presented MIMO, SIMO-LC and SIMO-SC systems, suffering from HWIs, experience insignificant performance gain in terms of average achievable rate with increasing space diversity. Interestingly, the increased transmit diversity fails to improve system performance for HWI-PGS. Whereas, the average rate performance of HWI-PGS improves but undergoes saturation with increasing receive diversity. Alternatively, IGS transmission significantly improves the system performance with increasing space diversity by effectively mitigating various HWIs. Moreover, it is observed that the gain obtained by increasing SNR with the receiver diversity is superior than that of transmitter diversity owing to the increased transmitter distortions/noise with increasing SNR.

\section{CONCLUSION}

In this paper, we proposed an accurate statistical model for communication links with non-ideal RF transceiver blocks,

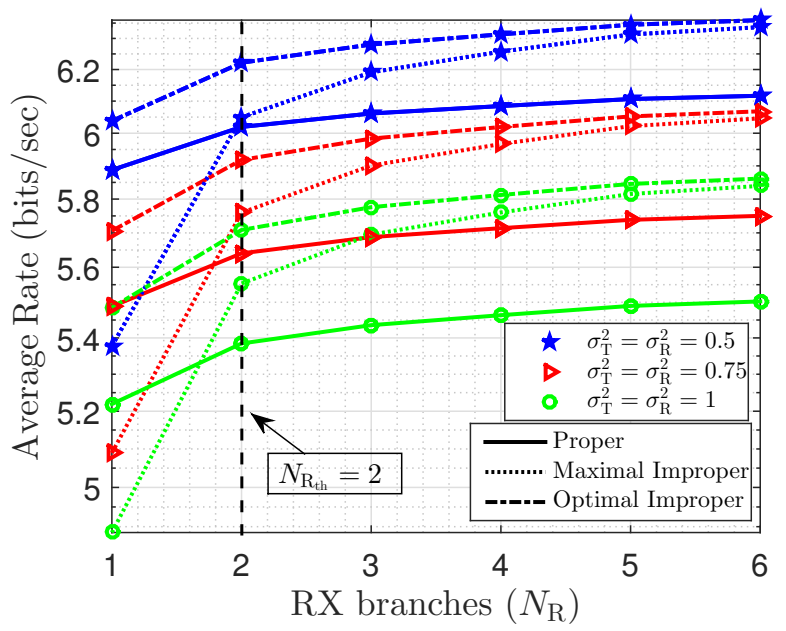

Fig. 11: SIMO-SC average achievable rate vs. $N_{\mathrm{R}}$ for various $\sigma_{\mathrm{T}}^{2}=\sigma_{\mathrm{R}}^{2}$

which captures the asymmetric property of the aggregated HWIs. The asymmetric signature of HWIs colored both the symmetric transmitted signal and the aggregate additive noise and converted them from PGS to IGS. The self-interference induced by the HWIs is compensated by employing IGS scheme at the transmitter side. Closed form expressions for the achievable rate are derived in case of ideal and nonideal hardware with PGS and IGS schemes. Throughout our study, we considered MIMO, SIMO-LC and SIMO-SC systems. Simulation results demonstrated the gain reaped by IGS scheme referring PGS scheme as a benchmark for HWI SIMO system. Furthermore, it was shown that the utility of IGS scheme in non-ideal scenario is overwhelming especially for higher impairment levels and higher data-rate communication. Therefore, IGS scheme is a suitable candidate to improve achievable rates with relatively less transmission power requirement, which makes it appropriate for the future generation of interference limited networks.

\section{APPENDiX A}

\section{PROOF OF LEMMA 2}

Proof. Given invertible $\mathbf{C}_{\mathbf{y}}$ and Schur complement, the determinant of augmented covariance matrix $\underline{\mathbf{C}}_{\mathbf{y}}$ is given as

$$
\left|\underline{\mathbf{C}}_{\mathbf{y}}\right|=\left|\begin{array}{cc}
\mathbf{C}_{\mathbf{y}} & \tilde{\mathbf{C}}_{\mathbf{y}} \\
\tilde{\mathbf{C}}_{\mathbf{y}}^{*} & \mathbf{C}_{\mathbf{y}}^{*}
\end{array}\right|=\left|\mathbf{C}_{\mathbf{y}}\right|\left|\mathbf{C}_{\mathbf{y}}^{*}-\tilde{\mathbf{C}}_{\mathbf{y}}^{*} \mathbf{C}_{\mathbf{y}}^{-1} \tilde{\mathbf{C}}_{\mathbf{y}}\right| \cdot
$$

Using the identity that $|\mathbf{Z}|=\left|\mathbf{Z}^{\mathrm{T}}\right|$, we obtain

$$
\left|\underline{\mathbf{C}}_{\mathbf{y}}\right|=\left|\mathbf{C}_{\mathbf{y}}\right|\left|\mathbf{C}_{\mathbf{y}}{ }^{\mathrm{H}}-\tilde{\mathbf{C}}_{\mathbf{y}}^{\mathrm{T}} \mathbf{C}_{\mathbf{y}}^{-\mathrm{T}} \tilde{\mathbf{C}}_{\mathbf{y}}^{\mathrm{H}}\right| .
$$

Using Definition 1 and the face that $\mathbf{C}_{\mathbf{y}}$ is Hermitian and $\tilde{\mathbf{C}}_{\mathbf{y}}$ is symmetric, we found

$$
\left|\underline{\mathbf{C}}_{\mathbf{y}}\right|=\left|\mathbf{C}_{\mathbf{y}}\right|\left|\mathbf{C}_{\mathbf{y}}-\tilde{\mathbf{C}}_{\mathbf{y}} \mathbf{C}_{\mathbf{y}}^{-\mathrm{T}} \tilde{\mathbf{C}}_{\mathbf{y}}^{\mathrm{H}}\right| .
$$

Eventually, using the determinant identities $\left|\mathbf{Z}^{-1}\right|=1 /|\mathbf{Z}|$, we obtain the desired form in (24). 


\section{REFERENCES}

[1] R. Q. Hu and Y. Qian, "An energy efficient and spectrum efficient wireless heterogeneous network framework for 5G systems," IEEE Commun. Mag., vol. 52, no. 5, pp. 94-101, May 2014.

[2] J. G. Andrews, S. Buzzi, W. Choi, S. V. Hanly, A. Lozano, A. C. Soong, and J. C. Zhang, "What will 5G be?" IEEE J. Sel. Areas Commun., vol. 32, no. 6, pp. 1065-1082, June 2014.

[3] A. Gupta and R. K. Jha, "A survey of 5G network: architecture and emerging technologies," IEEE access, vol. 3, pp. 1206-1232, July 2015.

[4] M. Dohler, T. Nakamura, A. Osseiran, J. F. Monserrat, O. Queseth, and P. Marsch, 5G Mobile and Wireless Communications Technology. Cambridge University Press, 2016.

[5] S. Buzzi, I. Chih-Lin, T. E. Klein, H. V. Poor, C. Yang, and A. Zappone, "A survey of energy-efficient techniques for $5 \mathrm{G}$ networks and challenges ahead," IEEE J. Sel. Areas Commun., vol. 34, no. 4, pp. 697-709, Apr. 2016.

[6] E. Hossain and M. Hasan, "5G cellular: key enabling technologies and research challenges," IEEE Trans. Instrum. Meas., vol. 18, no. 3, pp. 11-21, May 2015

[7] H. Mehrpouyan, M. Matthaiou, R. Wang, G. K. Karagiannidis, and Y. Hua, "Hybrid millimeter-wave systems: A novel paradigm for HetNets," IEEE Commun. Mag., vol. 53, no. 1, pp. 216-221, Jan. 2015.

[8] R. Krishnan, On the Impact of Phase Noise in Communication SystemsPerformance Analysis and Algorithms. Chalmers University of Technology, Apr. 2015.

[9] T. Schenk, $R F$ imperfections in high-rate wireless systems: impact and digital compensation. Springer Science \& Business Media, 2008.

[10] A.-A. A. Boulogeorgos, V. M. Kapinas, R. Schober, and G. K. Karagiannidis, "I/Q-imbalance self-interference coordination," IEEE Trans. Wireless Commun., vol. 15, no. 6, pp. 4157-4170, June 2016.

[11] X. Xia, D. Zhang, K. Xu, W. Ma, and Y. Xu, "Hardware impairments aware transceiver for full-duplex massive MIMO relaying," IEEE Trans. Signal Process., vol. 63, no. 24, pp. 6565-6580, Dec. 2015.

[12] M. Marey, M. Samir, and O. A. Dobre, "EM-based joint channel estimation and IQ imbalances for OFDM systems," IEEE Trans. Broadcast., vol. 58, no. 1, pp. 106-113, Mar. 2012.

[13] X. Zhang, M. Matthaiou, E. Björnson, M. Coldrey, and M. Debbah, "On the MIMO capacity with residual transceiver hardware impairments," in IEEE Int. Conf. Commun. (ICC), Sydney, June 2014, pp. 5299-5305.

[14] M. Mokhtar, A. Gomaa, and N. Al-Dhahir, "OFDM AF relaying under I/Q imbalance: Performance analysis and baseband compensation," IEEE Trans. Commun., vol. 61, no. 4, pp. 1304-1313, Apr. 2013.

[15] M. Mokhtar, A.-A. A. Boulogeorgos, G. K. Karagiannidis, and N. AlDhahir, "OFDM opportunistic relaying under joint transmit/receive I/Q imbalance," IEEE Trans. Commun., vol. 62, no. 5, pp. 1458-1468, May 2014.

[16] A.-A. A. Boulogeorgos, P. C. Sofotasios, B. Selim, S. Muhaidat, G. K. Karagiannidis, and M. Valkama, "Effects of RF impairments in communications over cascaded fading channels," IEEE Trans. Veh. Technol., vol. 65, no. 11, pp. 8878-8894, Jan. 2016.

[17] J. Qi and S. Aissa, "Analysis and compensation of I/Q imbalance in MIMO transmit-receive diversity systems," IEEE Trans. Commun., vol. 58, no. 5, May 2010.

[18] E. Björnson, M. Matthaiou, and M. Debbah, "A new look at dual-hop relaying: Performance limits with hardware impairments," IEEE Trans. Commun., vol. 61, no. 11, pp. 4512-4525, Nov. 2013.

[19] E. Björnson, J.Hoydis, M.Kountouris, and M. Debbah, "Hardware impairments in large-scale MISO systems: Energy efficiency, estimation, and capacity limits," in Proc. 18th Intern. Conf. Digital Signal Process. Santorini, Oct. 2013, pp. 1-6.

[20] E. Björnson, J. Hoydis, M. Kountouris, and M. Debbah, "Massive MIMO systems with non-ideal hardware: Energy efficiency, estimation, and capacity limits," IEEE Trans. Inf. Theory, vol. 60, no. 11, pp. 7112 7139, Sep. 2014.

[21] E. Björnson, P. Zetterberg, M. Bengtsson, and B. Ottersten, "Capacity limits and multiplexing gains of MIMO channels with transceiver impairments," IEEE Commun. Lett., vol. 17, no. 1, pp. 91-94, Jan. 2013.

[22] C. Studer, M. Wenk, and A. Burg, "MIMO transmission with residual transmit-RF impairments," in Int. ITG Workshop on Smart Antennas (WSA), Bremen, Feb. 2010, pp. 189-196.

[23] A.-A. A. Boulogeorgos, N. D. Chatzidiamantis, and G. K. Karagiannidis, "Energy detection spectrum sensing under RF imperfections," IEEE Trans. Commun., vol. 64, no. 7, pp. 2754-2766, July 2016.

[24] G. Zheng, I. Krikidis, C. Masouros, S. Timotheou, D.-A. Toumpakaris, and Z. Ding, "Rethinking the role of interference in wireless networks," IEEE Commun. Mag., vol. 52, no. 11, pp. 152-158, Nov. 2014.
[25] M. T. Kabir, M. R. Khandaker, and C. Masouros, "Interference exploitation in full duplex communications: Trading interference power for both uplink and downlink power savings," arXiv:1703.10666, Mar. 2017.

[26] P. J. Schreier and L. L. Scharf, Statistical signal processing of complexvalued data: the theory of improper and noncircular signals. Cambridge University Press, 2010.

[27] F. D. Neeser and J. L. Massey, "Proper complex random processes with applications to information theory," IEEE Trans. Inf. Theory, vol. 39, no. 4, pp. 1293-1302, July 1993.

[28] Y. Zeng, C. M. Yetis, E. Gunawan, Y. L. Guan, and R. Zhang, "Transmit optimization with improper Gaussian signaling for interference channels," IEEE Trans. Signal Process., vol. 61, no. 11, pp. 2899-2913, June 2013.

[29] O. Amin, W. Abediseid, and M.-S. Alouini, "Underlay cognitive radio systems with improper Gaussian signaling: Outage performance analysis," IEEE Trans. Wireless Commun., vol. 15, no. 7, July 2016.

[30] O.Amin, W. Abediseid, and M.-S. Alouini, "Overlay spectrum sharing using improper Gaussian signaling," IEEE J. Sel. Areas Commun., vol. 35, no. 1, pp. 50-62, Jan. 2017.

[31] S. Javed, O. Amin, and M.-S. Alouini, "Full-duplex relaying under I/Q imbalance using improper Gaussian signaling," in Intern. Conf. Acoust. , Speech, and Signal Process. (ICASSP 2017), New Orleans, Mar. 2017, pp. 6538-6542.

[32] M. Gaafar, O. Amin, A. Ikhlef, A. Chaaban, and M.-S. Alouini, "On alternate relaying with improper Gaussian signaling," IEEE Commun. Lett., vol. 20, no. 8, pp. 1683-1686, Aug. 2016.

[33] S. Javed, O. Amin, S. S. Ikki, and M.-S. Alouini, "Asymmetric hardware distortions in receive diversity systems: Outage performance analysis," IEEE Access, vol. 5, pp. 4492-4504, Feb. 2017.

[34] S.Javed, O. Amin, S. S. Ikki, and M.-S. Alouini, "On the achievable rate of hardware-impaired transceiver systems," in in Proc. IEEE Global Commun. Conf. (GLOBECOM), Singapore, Dec. 2017.

[35] E. Ollila, "On the circularity of a complex random variable," IEEE Signal Process. Lett., vol. 15, pp. 841-844, Nov. 2008.

[36] J. Li, M. Matthaiou, and T. Svensson, "I/Q imbalance in AF dual-hop relaying: Performance analysis in Nakagami-m fading," IEEE Trans. Commun., vol. 62, no. 3, pp. 836-847, Mar. 2014.

[37] E. Soujeri and G. Kaddoum, "The impact of antenna switching time on spatial modulation," IEEE Trans. Commun., vol. 5, no. 3, pp. 256-259, June 2016

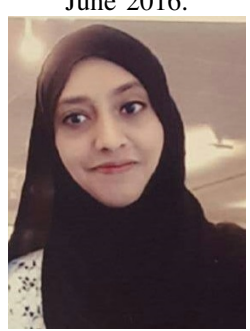

Sidrah Javed (S'16) received the B.E. degree in Electrical (Telecommunication) Engineering from National University of Science and Technology (NUST), Islamabad, Pakistan, in 2012. From 2012 to 2015, she has worked as a Research Engineer in National Radio and Telecommunication Corporation, Pakistan. She is currently pursuing M.S./Ph.D. degree at the Computer, Electrical and Mathematical Sciences \& Engineering (CEMSE) Division, King Abdullah University of Science and Technology (KAUST). Her research interests include hardwareconstrained communications, interference management, cooperative communications, vehicular tracking/monitoring and solar energy harvesting.

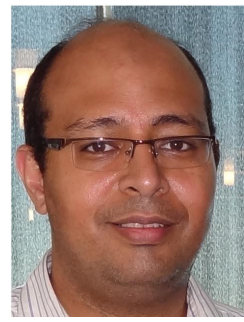

Osama Amin (S'07, M'11, SM'15) received the B.Sc. degree in electrical and electronics engineering from Aswan University, Aswan, Egypt, in 2000, the M.Sc. degree in electrical engineering from Assiut University, Assiut, Egypt, in 2004, and the $\mathrm{Ph} . \mathrm{D}$. degree in electrical and computer engineering from the University of Waterloo, Canada, in 2010. In 2012, he joined the Electrical and Electronics Engineering Department, Assiut University, as an Assistant Professor. He is currently a Research Scientist with the King Abdullah University of Science and Technology, Thuwal, Saudi Arabia. His general research interests lie in communications systems and signal processing for communications with special emphasis on wireless applications, optical wireless communication, molecular communications, terahertz communications, green communications, and cognitive radio.

Dr. Amin has served as a technical program committee (TPC) member for ICC, GLOBECOM, IEEE VTC, CROWNCOM, PIMRC, and ISSPIT conferences. He has served also as a Co-Organizer and a Co-Chair of the Next Generation Green ICT and 5G Networking 2015 at the IEEE International Conference on Ubiquitous Wireless Broadband in Montreal, Canada. In 2018, he served as the chair of the IoT, M2M, Sensor Networks, and Ad-Hoc Networking track in the VTC2018-fall held in Chicago, USA. He serves as an Associate Editor for the IEEE COMMUNICATIONS LETTERS. 


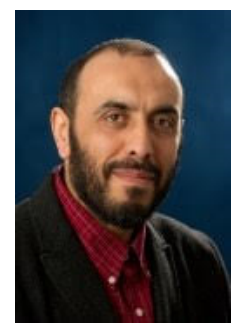

Salama Ikki received the B.S. degree from Al-Isra University, Amman, Jordan, in 1996, the M.Sc. degree from the Arab Academy for Science and Technology and Maritime Transport, Alexandria, Egypt, in 2002, and the Ph.D. degree from the Memorial University of Newfoundland, St. Johns, in 2009, all in electrical engineering. He is currently an Associate Professor in Wireless Communications with the Department of Electrical Engineering, Lakehead University. He was a Research Assistant with the INRS, University of Quebec, Montreal, from 2010 to 2012 and a Post-Doctoral Fellow with the University of Waterloo, Waterloo, ON, Canada, from 2009 to 2010.

He has authored or coauthored over 100 papers in peer reviewed IEEE international journals and conferences with over 2500 citations and has a current $\mathrm{H}$-index of 30 . He has been carrying out research in communications and signal processing for over 10 years. He was a recipient of the Best Paper Award published in the EURASIP Journal on Advances in Signal Processing, the IEEE Communications Letters and the IEEE Wireless Communications Letter Exemplary Reviewer Certificate in 2012, and the Top Reviewer
Certificate from the IEEE Transactions on Vehicular Technology in 2015. His Ph.D. student was awarded the second place for Best Poster from the School of Electrical and Electronic Engineering, Newcastle University, U.K., Annual Research Conference, in 2014. He serves on the Editorial Board of the IEEE Communications Letters and the IET Communications Proceeding. $\mathrm{He}$ is widely recognized as an expert in the field of wireless communications.

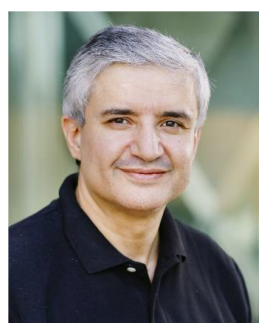

Mohamed-Slim Alouini (S'94, M'98, SM'03, F'09) was born in Tunis, Tunisia. He received the $\mathrm{Ph} . \mathrm{D}$. degree in Electrical Engineering from the California Institute of Technology (Caltech), Pasadena, CA, USA, in 1998. He served as a faculty member in the University of Minnesota, Minneapolis, MN, USA, then in the Texas A\&M University at Qatar, Education City, Doha, Qatar before joining King Abdullah University of Science and Technology (KAUST), Thuwal, Makkah Province, Saudi Arabia as a Professor of Electrical Engineering in 2009. His current research interests include the modeling, design, and performance analysis of wireless communication systems. 